Journal for ImmunoTherapy of Cancer

\title{
Aged neutrophils form mitochondria- dependent vital NETs to promote breast cancer lung metastasis
}

Chenghui Yang, ${ }^{1,2,3,4}$ Zhen Wang, ${ }^{1,2,3}$ Lili Li, ${ }^{1,2,5}$ Zhigang Zhang, ${ }^{1,6}$ Xiaoyan Jin, ${ }^{1,7}$ Pin Wu, ${ }^{1,8}$ Shanshan Sun, ${ }^{1,2,3}$ Jun Pan, ${ }^{1,2,3}$ Ke Su, ${ }^{1,2,3}$ Fang Jia, ${ }^{1,2,3}$ Leyi Zhang, ${ }^{1,2,3}$ Haijun Wang, ${ }^{9}$ Xiuyan Yu, ${ }^{1,2}$ Xuan Shao, ${ }^{1,2}$ Ke Wang, ${ }^{1,2}$ Fuming Qiu, ${ }^{1,5}$ Jun Yan, ${ }^{10}$ Jian Huang (1) 1,2,3

\section{ABSTRACT} et al. Aged neutrophils form mitochondria-dependent vital NETs to promote breast cancer lung metastasis. Journal for ImmunoTherapy of Cancer 2021;9:e002875. doi:10.1136/ jitc-2021-002875

- Additional supplemental material is published online only. To view, please visit the journal online (http://dx.doi.org/10. 1136/jitc-2021-002875).

CY, ZW and LL contributed equally.

Accepted 23 September 2021
Check for updates

(C) Author(s) (or their employer(s)) 2021. Re-use permitted under CC BY-NC. No commercial re-use. See rights and permissions. Published by BMJ.

For numbered affiliations see end of article.

Correspondence to

Dr Jian Huang;

drhuangjian@zju.edu.cn
Background Neutrophils-linked premetastatic niche plays a key role in tumor metastasis, but not much is known about the heterogeneity and diverse role of neutrophils in niche formation. Our study focuses on the existence and biological function of a rarely delved subset of neutrophils, named as tumor-associated aged neutrophils (Naged, CXCR4 ${ }^{+} \mathrm{CD}_{2} \mathrm{~L}^{\text {low }}$ ), involved in premetastatic niche formation during breast cancer metastasis.

Methods We explored the distributions of Naged in 206 patients and mice models (4T1 and MMTVPyMT) by flow cytometry. The ability of Naged to form neutrophil extracellular traps (NETs) and promote tumor metastasis in patients and mice was determined by polychromatic immunohistochemistry, scanning electron microscopy and real-time video detection. Furthermore, the differences among tumor-associated Naged, NonNaged and inflammation-associated aged neutrophils were compared by transcriptome, the biological characteristics of Naged were comprehensively analyzed from the perspectives of morphology, the metabolic capacity and mitochondrial function were investigated by Seahorse, co-immunoprecipitation (Co-IP), chromatin immunoprecipitation (ChIP) and transmission electron microscopy (TEM). Finally, 120 patients' sample were applied to confirm the acceleration of Naged formation through secreted NAMPT, and the importance of blocking this pathway in mice was evaluated.

Results We find that Naged accumulate in the lung premetastatic niche at early stage of breast tumorigenesis in multiple mice models and also exist in peripheral blood and metastatic lung of patients with breast cancer. Naged exhibit distinct cell marker and morphological feature of oversegmented nuclei. Further transcriptome reveals that Naged are completely different from those of NonAged or inflammation-associated aged neutrophils and illustrates that the key transcription factor SIRT1 in Naged is the core to maintain their lifespan via mitophagy for their function. The responsible mechanism is that SIRT1 can induce the opening of mitochondrial permeability transition pore channels to release mitochondrial DNA and lead to the mitochondria-dependent vital NETs formation, rather than traditional Cit-Histone $\mathrm{H} 3$ dependent fatal-NETs. Further mechanically investigation found tumor derived NAMPT could induce Naged formation. Additionally, therapeutic interventions of Naged and its formation-linked pathways could effectively decrease breast cancer lung metastasis.

Conclusions Naged exerts a vital role in breast cancer lung metastasis, and strategies targeting SIRT1-NagedNETs axis show promise for translational application.

\section{BACKGROUND}

Metastasis is the leading cause of death in patients with cancer. Distant organs undergo necessary changes to create a local microenvironment suitable for tumor cell colonization, called the 'premetastatic niche', which has been deemed a pivotal stage of tumor metastasis. ${ }^{1-3}$ Currently, the formation of the premetastatic niche is postulated to be driven by primary tumors and involves stromal elements. ${ }^{1-8}$ Among these components, neutrophils have been shown to play a key role in premetastatic niche formation. ${ }^{9-12}$ The antitumor and protumor effects of neutrophils have long been a topic of discussion. ${ }^{9}{ }^{13}$ Neutrophils are classified into N1-like/N2-like neutrophils according to their functional properties and lowdensity neutrophils (LDNs)/high-density neutrophils (HDNs) according to their cell density. ${ }^{14-16}$ Subsequently, various new subsets of tumor-infiltrating neutrophils and novel functions have continued to be discovered.

Neutrophil extracellular traps (NETs) are unique extracellular network structures that are produced by neutrophils and consist of DNA, histones and granular contents. NETs are first discovered in infectious inflammation, exerting a bactericidal effect via antibacterial granules. ${ }^{17}$ NETs have also been found to play important roles in aseptic inflammatory diseases such as gout, cystic fibrosis, type 1 diabetes, rheumatoid arthritis, pre-eclampsia and tumors. ${ }^{18}$ Previous studies found that NETs exerted antitumor effects. ${ }^{19}$ However, subsequent studies revealed that 
NETs enhance tumor progression by affecting the endothelium, platelets, and the extracellular matrix and even changing the tumor cells themselves. ${ }^{20-25}$

As the energy factory of the cell, mitochondria are important organelles for cells to maintain their homeostasis and function. But, mitochondria have long been neglected in neutrophil research because neutrophils are generally regarded as oligomitochondrial cells. ${ }^{26}$ Since 2004, the role of mitochondria in the apoptosis of neutrophils mediated by the Caspase pathway has been studied extensively. ${ }^{27-29}$ According to a subsequent study, immature c-Kit ${ }^{+}$neutrophils rely on oxidative phosphorylation (OXPHOS).$^{30}$ Mitochondria have also been reported to participate in neutrophil differentiation, maturation and activation. ${ }^{31-34}$ Furthermore, the chemotaxis and polarization of neutrophils are also thought to involve mitochondria. ${ }^{35}$ Recently, the role of mitochondria in NETs began to be discovered in lupus-like diseases. ${ }^{37} 38$ However, the role of mitochondria in neutrophils located in the tumor premetastatic niche remains unclear.

In our study, we found that a group of aged neutrophils $\left(\mathrm{CXCR}^{+}{ }^{+} \mathrm{CD} 62 \mathrm{~L}^{\text {low }}\right)$ accumulated in the lung premetastatic niche of breast cancer and captured tumor cells by generating vital NETs. Further mechanistic studies revealed that vital NETs formation depended on SIRT1 activation in neutrophils via NAMPT secreted by the primary tumor. This novel mechanism of vital NETs was regulated by mitochondrial DNA. Strategies targeting the aged neutrophil-SIRT1-vital NET pathway may effectively inhibit the lung metastasis of breast cancer and provide new ideas for tumor treatment.

\section{MATERIALS AND METHODS \\ Specimen acquisition and processing}

The heparin-anticoagulated human peripheral blood (PB) sample was centrifuged to separate blood cells from plasma. Blood cells were then lysed with lysis buffer for 15 min (BD Biosciences, \#349202) to remove erythrocytes and washed twice with phosphate buffered saline (PBS) and resuspended in PBS for uses in subsequent experiments.

Mouse specimens were processed at 9:00 hours to prevent the effect of the physiological circadian rhythm. ${ }^{39}$ Bone marrow cells (BMCs) were obtained from the tibia and femur and impurities were subsequently removed using a $40 \mu \mathrm{m}$ cell strainer (BD FALCON, \#352340). PB was collected from the retro-orbital vein and then lysed for $15 \mathrm{~min}$. A single cell suspension of primary tumor and lung tissues was obtained by mincing and digesting in serum-free RPMI-1640 medium containing $1 \mathrm{mg} / \mathrm{mL}$ collagenase IV (Sigma-Aldrich, \#V900893) in a $37^{\circ} \mathrm{C}$ shaking incubator for 2 hours, followed by filtration through a $40 \mu \mathrm{m}$ cell strainer.

\section{Flow cytometry and fluorescence-activated cell sorting}

For cell surface marker staining, the cell suspension was incubated with FcR Blocking Reagent (BioLegend,
\#101319 for mice sample and \#422301 for human sample) followed by incubation with a mixture of fluorochrome-conjugated anti-mouse monoclonal Abs specific for CD45 (Clone 30-F11, \#103116), CD11b (Clone M1/70, \#101216), Ly-6G (Clone 1A8, \#127614 and \#127606), CD62L (Clone MEL-14,\#104408), CXCR4 (Clone L276F12, \#146507), CD3e (Clone 145-2C11, \#100320), CD4 (Clone RM4-5, \#100510), CD8 (Clone 53-6.7, \#100734), CD19 (Clone 6D5, \#115508), CD49b (Clone DX5,.\#108910), and F4/80 (Clone BM8, \#123110) or anti-human monoclonal Abs specific for CD45 (Clone HI30, \#304014), CD11b (Clone ICRF44, \#301322), CD66b (Clone G10F5, \#305104), CD62L (Clone DREG-56, \#304806), and CXCR4 (Clone 12G5; \#306510, all from BioLegend). Samples were incubated in the dark for $20 \mathrm{~min}$ at $4^{\circ} \mathrm{C}$, and washed twice with cell staining buffer (Biolegend, \#420201). For intracellular staining, cells were fixed and permeabilized using the Fixation and Permeabilization Solution (BD Biosciences, \#554722). Subsequently, cells were stained with fluorophore-conjugated antibodies specific for interferon $\gamma$ (IFN- $\gamma$ ) (BioLegend, Clone XMG1.2, \#505850) and interleukin 2 (IL-2) (BioLegend, Clone JES6-5H4, \#503808) and incubated in the dark for $30 \mathrm{~min}$ at $4^{\circ} \mathrm{C}$. For intranuclear staining, The True-Nuclear Transcription Factor Buffer Set (BioLegend, \#424401) was used to label transcription factors according to the manufacturer's instructions. Then, mAbs specific for KIRF8 (eBioscience, Clone V3GYWCH, \#12-9852-82), phospho-STAT3 (eBioscience, Clone LUVNKLA, \#12-9033-42), phosphoSTAT5 (eBioscience, Clone SRBCZX, \#12-9010-42), and ROR gamma (t) (eBioscience, Clone AFKJS-9, \#12-698882) were added and incubated in the dark for at least 30 min at room temperature (RT).

Data were acquired with a FACSCanto II flow cytometer (BD Biosciences, USA) and analyzed with FlowJo software (V.10.0 for Windows, Tree Star).

For fluorescence-activated cell sorting (FACS), the single cell suspension was sorted with a FACSAria II cell sorter (BD Biosciences, USA).

\section{Magnetic isolation of neutrophils}

Mouse neutrophils were isolated by Mouse Neutrophil Isolation Kit (Miltenyi, \#130-097-658). Briefly, 50 $\mathrm{LL}$ of the Neutrophil Biotin-Antibody Cocktail were added to $200 \mu \mathrm{L}$ of a cell suspension $\left(5 \times 10^{7}\right.$ total cells in MagneticActivated Cell Sorting (MACS) buffer) and incubated for $15 \mathrm{~min}$ at $4^{\circ} \mathrm{C}$. After washes with MACS buffer two times, $100 \mu \mathrm{L}$ of Anti-Biotin MicroBeads were added to $400 \mu \mathrm{L}$ of the cell suspension. An LS column and MidiMACS separator (Miltenyi) were applied for subsequent magnetic sorting.

Human PB-derived neutrophils were isolated by EasySep Direct Human Neutrophil Isolation Kit (STEMCELL, \#19666). The isolation antibody cocktail $(50 \mu \mathrm{L} / 1 \mathrm{~mL}$ whole blood) and RapidSpheres beads $(50 \mu \mathrm{L} / 1 \mathrm{~mL}$ whole blood) were added to whole blood and incubated for $5 \mathrm{~min}$ at RT successively. Then, isolation buffer was 
added to the cell suspension to bring the volume to $10 \mathrm{~mL}$, and the tube was placed in the EasySep Magnets (STEMCELL Technologies) for $5 \mathrm{~min}$. The enriched cell suspension was collected in a new tube and incubated with RapidSpheres beads $(50 \mu \mathrm{L} / \mathrm{mL})$ for another $5 \mathrm{~min}$. A second separation with EasySep Magnets for $5 \mathrm{~min}$ was performed, and the enriched cell suspension was collected for subsequent in vitro induction.

\section{Measurement of neutrophil bioenergetics}

Neutrophil adherence was achieved by plating sorted neutrophils in Seahorse assay media containing $2 \mathrm{mM}$ glutamine and $25 \mathrm{mM}$ glucose and spinning at the lowest acceleration to $45 \times \mathrm{g}$ followed by natural deceleration. Lung aged and non-aged neutrophils obtained by FACS, primary control neutrophils and SRT1720-induced neutrophils were seeded at a density of $0.3 \times 10^{6}$ cells per well and incubated for $40 \mathrm{~min}$ at $37^{\circ} \mathrm{C}$ without $\mathrm{CO}_{2}$. The oxygen consumption rate (OCR) (Agilent Technologies, \#1 03275-100) and glycolysis rate (Agilent Technologies, \#1 03344-100) were analyzed at $37^{\circ} \mathrm{C}$ without $\mathrm{CO}_{2}$ using the Seahorse XFe96 Analyzers (Agilent Technologies, CA) according to the manufacturer's instructions. Port additions and times were performed as indicated in the figure.

\section{RESULTS \\ Naged accumulate in the lung premetastatic niche at early stage of breast tumorigenesis, promoting lung metastasis}

In an orthotopic inoculation model using 4T1 tumor cells, the percentages of the major infiltrating immune cell subsets in the lung tissue were reduced during tumor progression, except for neutrophils (figure 1A and online supplemental figure 1A,B). Similar results were also obtained from the MMTV-PyMT model of spontaneous breast cancer (online supplemental figure 1C). Considering premetastatic niche formation is an early stage of tumor metastasis, the Ly-6G mAb was administered at different tumor stages (online supplemental figure 1D,E). In both models, the early depletion of neutrophils effectively reduced lung metastasis, but a late intervention was ineffective (figure 1B). The nucleus morphology of lung-infiltrating neutrophils was a combination of round, horseshoe and lobulated shapes, but the cells mainly exhibited a lobulated shape during the period of niche formation (figure $1 \mathrm{C}$ and $\mathrm{D}$ and online supplemental figure $1 \mathrm{~F}$ ). Lobulated neutrophils were first defined as aged neutrophils $\left(\mathrm{CD} 45^{+} \mathrm{CD} 11 \mathrm{~b}^{+} \mathrm{Ly}-6 \mathrm{G}^{+} \mathrm{CXCR} 4^{+} \mathrm{CD} 62 \mathrm{~L}^{\text {lo }}\right.$, Naged) in inflammation in 2013. ${ }^{39}$ The percentage of Naged was significantly increased in the PB and lung in the 4T1 model and MMTV-PyMT model (figure 1E-G and online supplemental figure $1 \mathrm{G}, \mathrm{H})$. We also detected neutrophils in the PB from patients with newly diagnosed breast cancer and found that both Naged and non-aged neutrophils were present (figure $1 \mathrm{H}$ ). Furthermore, patients with breast cancer, particularly triple negative breast cancer (TNBC), had higher proportion of Naged in their PB than patients with breast fibroadenoma, after excluding the influence of patients' age (figure 1I and J and online supplemental figure $2 \mathrm{~A}-\mathrm{D}$ ).

\section{Naged promote lung metastasis by capturing tumor cells via NETs}

NETs were classic mechanisms for killing harmful microorganisms, but Naged did not affect the proliferation of tumor cells nor did they kill tumor cells (online supplemental figure 2E,F). Instead, Naged captured tumor cells though NETs in vivo and in vitro (figure 2A,B). Furthermore, we showed in a video how Naged capture tumor cells (online supplemental movie 1,2).

Moreover, Naged were in direct contact with tumor cells through NETs in the mouse metastatic lung tumors (figure 2C). Lung metastatic lesions from patients with breast cancer showed that Naged formed more NETs than normal lung tissues (figure 2D-F and online supplemental figure $2 \mathrm{G}$ ). An in vivo imaging study was performed to further show that the Naged captured tumor cells and promoted their retention in the lung (online supplemental figure $2 \mathrm{H}, \mathrm{I}$ ), and the results revealed a significantly weaker fluorescence intensity in the lungs of naive mice than in the lungs of tumor-bearing mice (online supplemental figure 2J). In addition, Naged and non-aged neutrophils were equally injected into tumorbearing mice, respectively. Then, luciferase-expressing tumor cells were injected into those two groups, and imaging revealed stronger fluorescence intensity in the Naged group (figure 2G). A similar phenomenon was also observed in nude mice, suggesting that the effect is independent of $\mathrm{T}$ cells.

\section{Naged are a unique subset of neutrophils}

Whole transcriptome sequencing of inflammationassociated aged neutrophils, tumor-associated aged neutrophils (Naged) and paired non-aged neutrophils from lung tissues was performed to further investigate the properties of these neutrophil subsets. The transcriptomes of these three groups of cells were completely distinct (figure 3A), while the results has been verified using RT-PCR (online supplemental figure 3A,B). Based on the functional cluster analysis, these three groups of neutrophils had their own unique transcriptomic characteristics (online supplemental figure 3C,D). Then, we focused on the differences between Naged and non-aged neutrophils derived from tumor-bearing mice (figure 3B). Further analysis revealed that Naged could not be classified as N1-like or N2-like tumor-associated neutrophils (figure 3C and online supplemental figure 3E). Given the strong immunomodulatory effect of Naged, we wondered about their association with classical polymorphonuclear myeloid-derived suppressor cells (PMN-MDSCs), which possess a potent immunosuppressive ability. The method used to separate neutrophils from PMN-MDSCs in tissue was gradient centrifugation..$^{40}$ PMN-MDSCs are enriched in areas of low density, whereas neutrophils are high-density cells. ${ }^{16}$ Consistent with the finding reported 


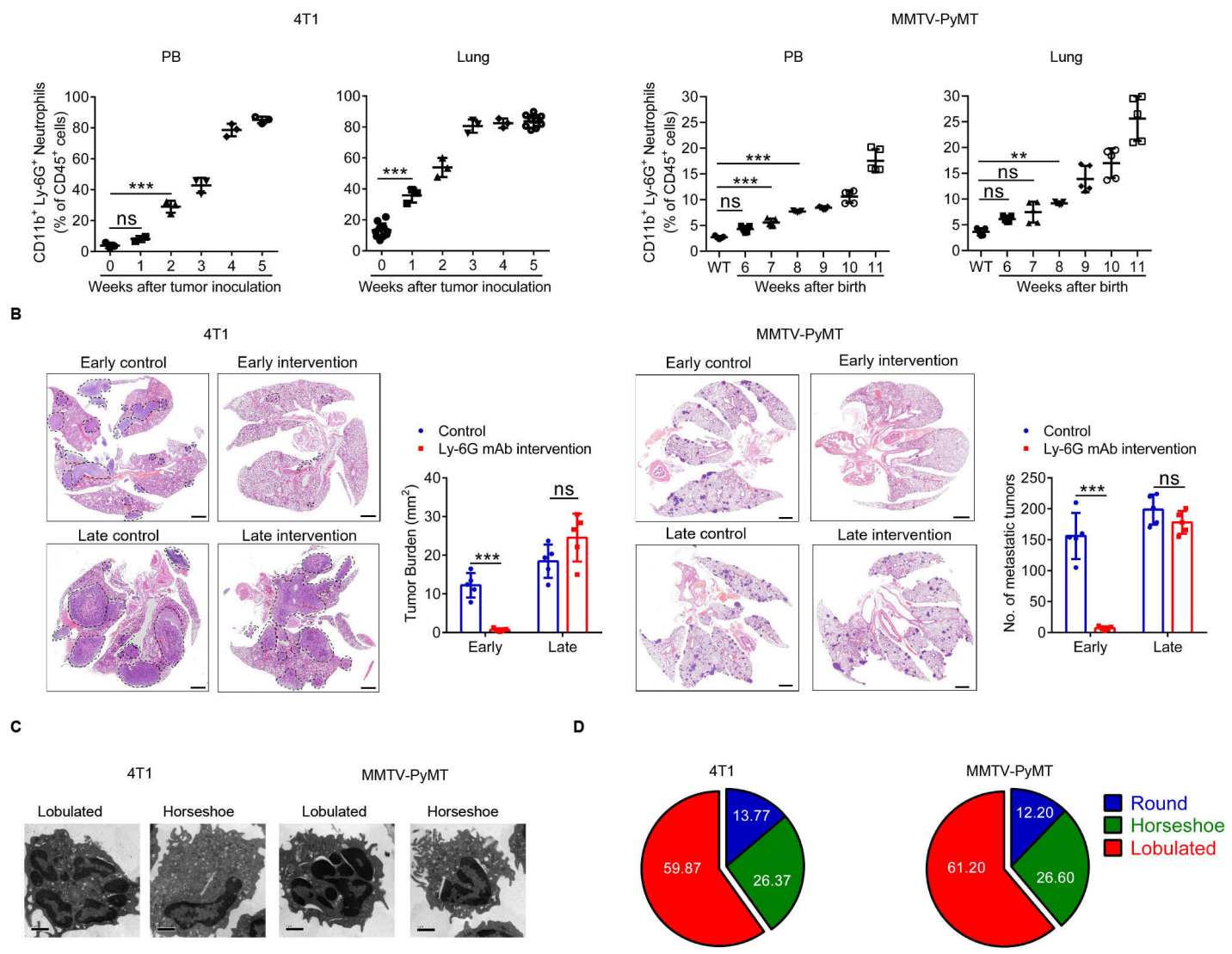

E

$\mathbf{F}$

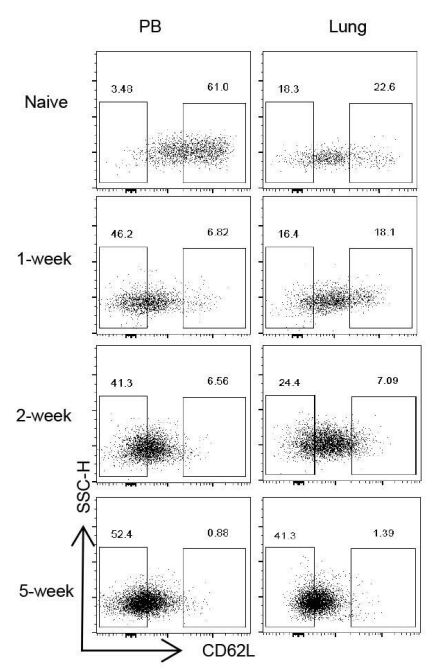

H

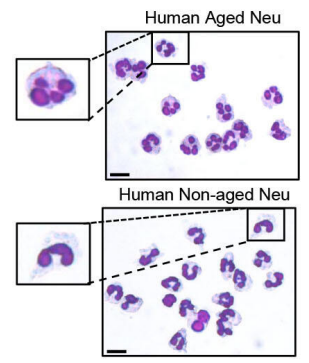

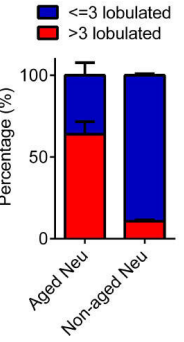

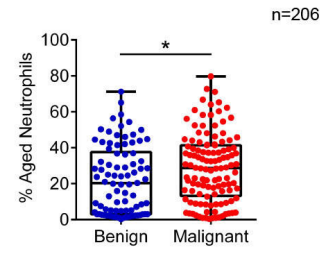

$n=206$

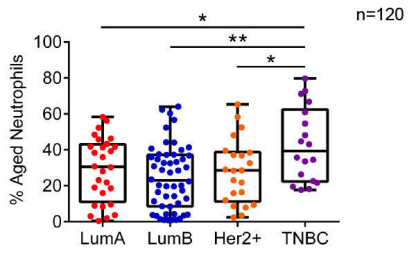

Figure 1 (Continued) 
Figure 1 Naged accumulate in the lung premetastatic niche at early stage of breast tumorigenesis, promoting lung metastasis. (A) Flow cytometry analysis of weekly dynamic changes of neutrophils in PB and lung from the 4T1 model (left panel) and MMTV-PyMT model (right panel). (B) H\&E staining and quantification of lung metastasis after treatment with or without the Ly$6 \mathrm{G} \mathrm{mAb}$ at different stages of tumor progression in the 4T1 model (left panel) and MMTV-PyMT model (right panel). Scale bar, $1 \mathrm{~mm}$. (C, D) Representative transmission electron microscopy (TEM) images (C) and quantification (D) of different neutrophil nucleus morphology in the lungs from 4T1 tumor-bearing mice at 2 weeks after tumor cell inoculation and 9-week-old MMTVPyMT mice determined using Giemsa staining. Scale bar, $1 \mu \mathrm{m}$. (E-G) Flow cytometry analysis (E) and quantification of weekly dynamic changes in PB and lung-infiltrating aged and non-aged neutrophils in the 4T1 model (F) and MMTV-PyMT model (G). (H) Representative images and quantification of Giemsa staining showing the nucleus morphology of neutrophils in PB from patients with breast cancer. Scale bar, $5 \mu \mathrm{m}$. (I) Quantification of aged neutrophils in PB from patients with breast fibroadenoma and breast cancer. (J) Quantification of aged neutrophils in PB from patients with different molecular types of breast cancer. Data are presented as the mean \pm SD from one representative experiment. Similar results were obtained from three independent experiments, unless indicated otherwise. Statistical analysis was performed by two-tailed unpaired Student's t-test (B), MannWhitney $U$ tests (I) and one-way ANOVA (A, F, G, J). ${ }^{*} p<0.05,{ }^{* *} p<0.01$, and ${ }^{\star * \star} p<0.001$. ANOVA, analysis of variance; ns, not significant; PB, peripheral blood; TNBC, triple negative breast cancer.

by Sagiv, LDNs had a larger FSC than HDNs. However, no significant differences in the proportion of Naged were observed between LDNs and HDNs (figure 3D and online supplemental figure $3 \mathrm{~F}$ ), suggesting that the classification of the aged/non-aged neutrophils was different from the density classification. Naged had a clear immunosuppressive function, but it was not as prominent as LDNs (figure 3E-G and online supplemental figure $3 \mathrm{G})$. In addition, according to the study by Dmitry, ${ }^{40}$ the biochemical and molecular parameters associated with characteristic MDSC functions were compared between LDNs and Naged (online supplemental table 1). Based on these data, Naged may have different roles from PMNMDSCs during tumor metastasis.

\section{The SIRT1-C/EBPE-Lamin B receptor axis regulates the oversegmentation of Naged}

The transcriptome sequencing results showed that the crucial transcription factor determining Naged was SIRT1 (figure 4A and B), and SIRT1 expression was upregulated in Naged from both mice and humans (figure 4C). Furthermore, the proportion of Naged increased with treatment with increasing concentrations of the commonly used SIRT1 agonist SRT1720 in vitro (figure 4D), ${ }^{41}$ and the neutrophil nucleus morphology changed from round to oversegmented (figure 4E). Previous research considered the nuclear membrane protein Lamin B receptor (LBR) could promote neutrophil nucleus lobulation. ${ }^{42}$ LBR was not only highly abundant in the Naged (figure 4F,G) but also regulated by SIRT1 (figure $4 \mathrm{H}$ and online supplemental figure $4 \mathrm{~A})$. In order to determine the effect of LBR on neutrophils, we attempted to knock out $L B R$ in BM-derived neutrophils. Prior to the experiment, BM neutrophils were pretreated with GM-CSF to extend their lifespan, which did not affect the expression of CD62L, the nucleus morphology of the cells, or LBR expression (online supplemental figure 4B-D). The LBR-knockout neutrophils did not respond to SRT1720, and the proportion of Naged remained steady (figure 4I, J and online supplemental figure $4 \mathrm{E}-\mathrm{G})$. C/EBPE was an important transcription factor that regulated LBR. ${ }^{43}$ However, SRT1720 did not affect the expression of C/EBPE but functioned as a deacetylase to reduce the level of $\mathrm{C} / \mathrm{EBP} \varepsilon$ acetylation (figure $4 \mathrm{~K}$ and online supplemental figure $4 \mathrm{H})$. More importantly, C/EBPE effectively bound the LBR promoter to promote LBR transcription (figure $4 \mathrm{~L}, \mathrm{M}$ ). Deacetylated C/EBPE more effectively bound the DNA at the LBR promoter, which might explain why SIRT1 could promote LBR expression and accelerate the formation of nuclear membrane folds.

\section{SIRT1 prolongs the survival of Naged through mitophagy}

Another important function of SIRT1 was to modulate cell metabolism. Both the OXPHOS (OCR) and glycolysis (glycolytic rate) levels of Naged were significantly reduced compared with non-aged neutrophils (figure 5A,B and online supplemental figure 5A), while SIRT1 mainly affected the OCR of neutrophils (figure 5C and online supplemental figure $5 \mathrm{~B}$ ). The change in the OCR was mainly related to mitochondrial function. Using transmission electron microscopy, we not only observed oversegmentation of the nucleus but also autophagic vesicles inside the Naged, while enriched and complete mitochondria were detected in non-aged neutrophils (figure 5D). Furthermore, the autophagic activity of Naged was significantly increased (figure 5E) and regulated by SRT1720 (figure 5F). Autophagy was divided into macroautophagy and microautophagy, and SIRT1 mainly regulated mitophagy in neutrophils (figure $5 \mathrm{G}, \mathrm{H}$ and online supplemental figure 5C,D). As predicted by the sequencing results, Naged had a longer lifespan than non-aged neutrophils (figure $5 \mathrm{I}, \mathrm{J}$ ), and the extended neutrophil longevity induced by SRT1720 was inhibited by Mdivi-1, an inhibitor of mitophagy (figure $5 \mathrm{~K}) .{ }^{44} \mathrm{In}$ summary, SIRT1 not only affects the mitochondrial energy metabolism of Naged but also prolongs the lifespan of the cells through mitophagy.

\section{SIRT1 induces Naged to form mitochondria-dependent vital NETs via opening mitochondrial permeability transition pore channels}

Using scanning electron microscopy, we found that Naged have the ability to form NETs (figure 6A), but the type of NET formed by these cells was different from the 
A

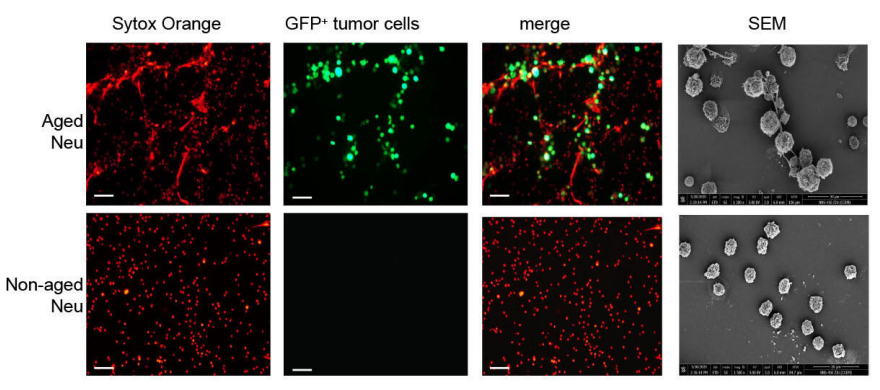

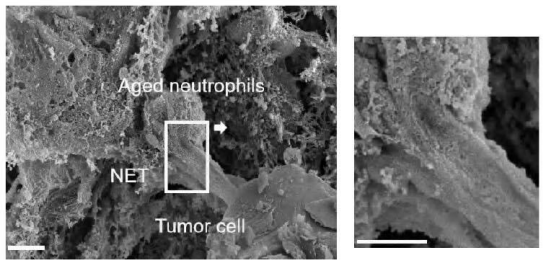

D

Figure 2 (Continued)
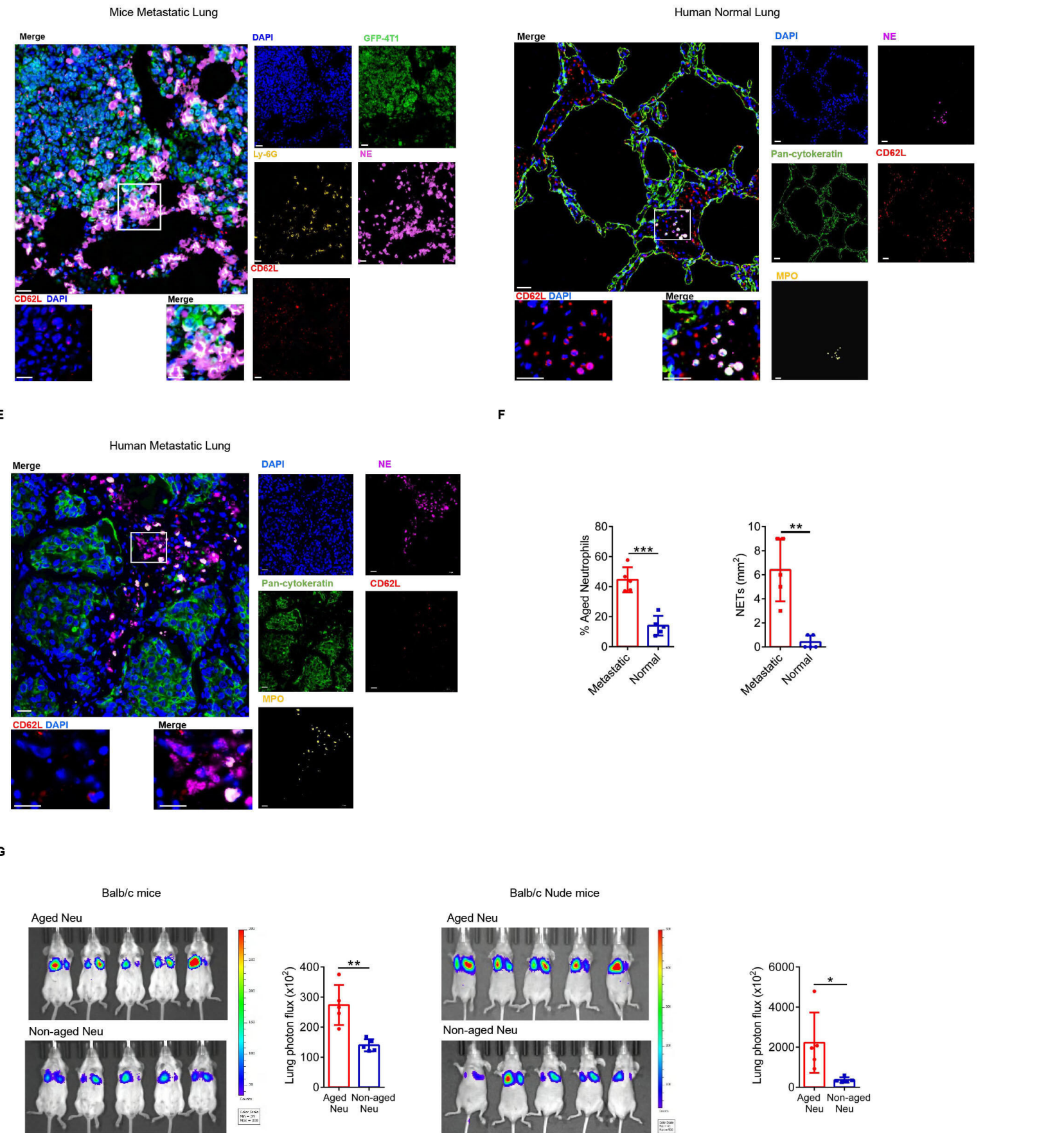
Figure 2 Naged promote lung metastasis by capturing tumor cells via NETs. (A) Images of immunofluorescence staining (left panel) and SEM (right panel) of NETs produced by aged and non-aged neutrophils in the lungs of 2 weeks tumor-bearing mice. Red, Sytox Orange. Green, GFP). Scale bar, $50 \mu \mathrm{m}$. (B) SEM images of aged neutrophils in the lungs of 2 weeks tumor-bearing mice. Scale bar, $2 \mu \mathrm{m}$. (C) Multiplex Immunohistochemistry analysis of lung metastasis in tumor-bearing mice showing the

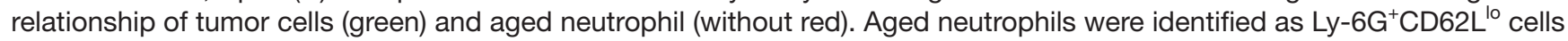
(lower left panel) and tumor cells were identified as GFP ${ }^{+}$cells. NETs were labeled with NE (neutrophil elastase). Scale bar, $10 \mu \mathrm{m}$. (D, E) Multiplex Immunohistochemistry analysis of relatively normal lung (D) and metastatic lung (E) tissues from patients with breast cancer showing the relationship of breast cancer cells (green) and aged neutrophils (without red). Aged neutrophils were identified as MPO ${ }^{+} \mathrm{CD}_{2} \mathrm{~L}^{\mathrm{lo}}$ cells (lower left panel). Tumor cells and pulmonary epithelial cells were identified as pancytokeratin $^{+}$cells. NETs were labeled with NE. Scale bar, $10 \mu \mathrm{m}$. (F) Quantification of aged neutrophils (left panel) and NETs (right panel) in relatively normal lung and metastatic lung tissues from patients with breast cancer. (G) Representative bioluminescent images and quantitative analysis of the lungs of 2 weeks tumor-bearing BALB/c mice (left panel) and nude mice (right panel) injected with luciferase-expressing 4T1 tumor cells after the injection of aged or non-aged neutrophils. Data are presented as the means $\pm S D$ from one representative experiment. Similar results were obtained from three independent experiments, unless indicated otherwise. Statistical analysis was performed by two-tailed unpaired Student's t-test $(F, G)$. ${ }^{*} p<0.05$, ${ }^{* *} p<0.01$, and ${ }^{\star \star \star} p<0.001$. GFP, green fluorescent protein; NETs, neutrophil extracellular traps; ns, not significant.

classic network structure in neutrophils. ${ }^{45}$ PMA induced the formation of the most classic type of NETs, and this process depended on the Nox-PAD4 signaling pathway. ${ }^{46}$ However, neither Nox nor PAD4 was activated during Naged NET formation (online supplemental figure 6A,B). Moreover, the effector protein Cit-HistoneH3 was not upregulated (online supplemental figure 6C). Therefore, we further observed the NETs induced by SRT1720 and PMA, respectively (figure 6B). The PMAinduced NETs were cloud-like, while SRT1720 induced the formation of needle-like NETs. Moreover, the Nox inhibitor apocynin suppressed the PMA-induced formation of NETs but not SRT1720-induced NET formation (online supplemental figure 6D). More importantly, the neutrophils were all dead after PMA induction, and their cell membranes were ruptured. After the SRT1720 intervention, the neutrophils remained intact, except for the formation of mitophagosomes (online supplemental figure 6E,F). Interestingly, SRT1720-induced NETs contained mitochondrial components but not CitHistoneH3, while PMA-induced NETs contained both of these components (figure 6C and online supplemental figure $6 \mathrm{G}$ ). A further examination of the DNA components of neutrophil NETs revealed that both SRT1720 and PMA increased the amount of cell-free dsDNA (cfDNA) in cultured supernatants (online supplemental figure $6 \mathrm{H})$; mitochondrial DNA dominated after SRT1720 induction, while nuclear DNA dominated after PMA induction (figure 6D and E). Therefore, we detected the pore channels of mitochondria on neutrophils and found that the mitochondrial permeability transition pore (mPTP), the large pore channels of mitochondria, were opened by SRT1720 (figure 6F), which was presumed to be the outlet of mitochondrial DNA. ${ }^{46}$ Ciclosporin A and TRO19622, used as mPTP inhibitors, inhibited SRT1720-induced NET formation and reduced the cfDNA content (figure 6G,H and online supplemental figure 6I). Thus, SIRT1 induces the formation of a new type of vital NET that depends on mitochondria, which is clearly different from PMA-induced NETs and do not depend on the PAD4 signaling pathway.

\section{Tumor-secreted NAMPT is responsible for the expression of SIRT1 on Naged, promoting premetastatic niche formation}

Neutrophils are mainly derived from BM hematopoietic stem cells (HSCs). In order to further prove the role of SIRT1 in regulating Naged in vivo, we transfected the HSCs with shSIRT1-1 lentivirus, of which the knock-down efficiency had been confirmed in vitro, and those shSIRT1HSCs were transplanted into irradiated recipient mice for reconstruction (online supplemental figure 7A,B). We found that the SIRT1 expression of BMCs was significantly declined in the shSIRT1-HSC transplantation (HSCT) group (online supplemental figure 7C,D). Neutrophils in the lung tissue of the 2-week tumor-bearing mice were decreased slightly in the shSIRT1 HSCT group (online supplemental figure 7E). More importantly, Naged were significantly reduced (figure 7A), along with the decrease of oversegmented nucleic neutrophils and less NETs formation (figure $7 \mathrm{~B}$ and $\mathrm{C}$ ). It was also found that the key proteins SIRT1, LBR, and LC3B-II expression of lunginfiltrating neutrophils in the shSIRT1 HSCT group were all downregulated,which explained the importance of the SIRT1-LBR-LC3B pathway (figure 7D). Furthermore, we observed that lung metastasis of the shSIRT1 HSCT group was significantly reduced, indicating that the BM-derived SIRT1 played an important role in regulating Naged and promoting breast cancer lung metastasis (figure $7 \mathrm{E}$ ).

NAMPT is a rate-limiting enzyme that converts nicotinamide into nicotinamide adenine dinucleotide $\left(\mathrm{NAD}^{+}\right)$, which in turn activates $\mathrm{NAD}^{+}$-dependent protein deacetylases called sirtuins that regulate multiple cellular functions. ${ }^{47}$ High expression of NAMPT was significantly associated with a poor prognosis of patients with breast cancer during an analysis of the TCGA database (figure 7F and online supplemental figure 7F). Therefore, we performed and analyzed immunohistochemical staining in samples from 100 patients with breast tumor and found 


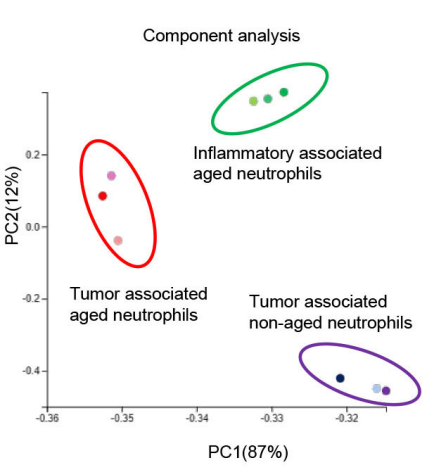

B
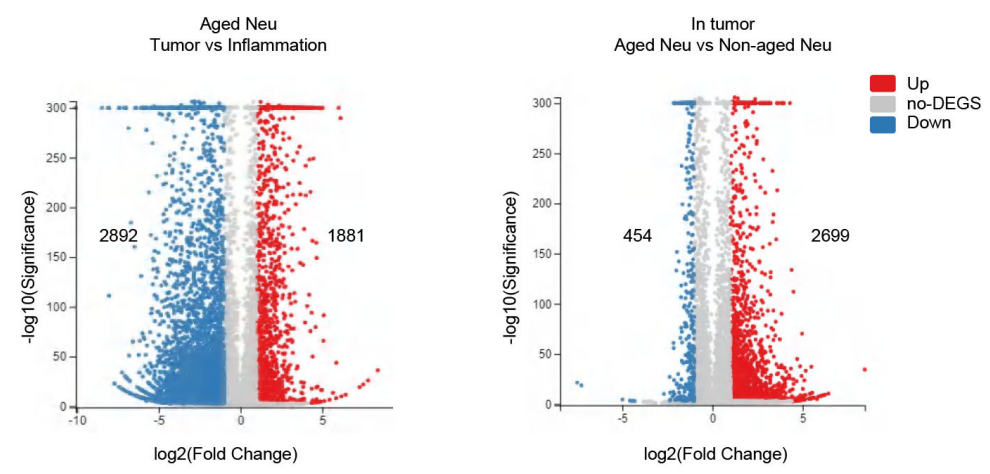

c

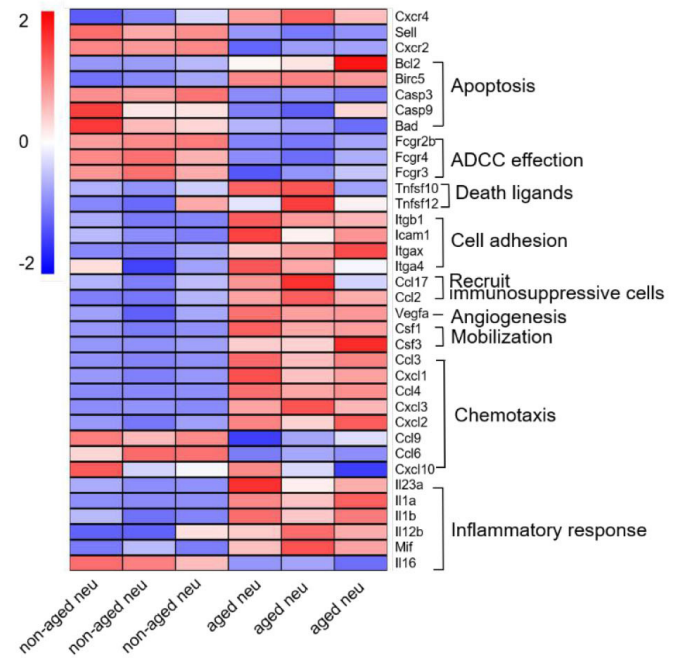

\begin{tabular}{|c|c|c|}
\hline & genes & change \\
\hline \multirow{9}{*}{$\begin{array}{l}\text { N1 like } \\
\text { genes }\end{array}$} & Tnf-a & $\uparrow$ \\
\hline & Icam-1 & $\uparrow$ \\
\hline & Fas & - \\
\hline & Ros & $\downarrow$ \\
\hline & $\mathrm{Ccl} 3$ & $\uparrow$ \\
\hline & iNos & $\downarrow$ \\
\hline & GranzymeB & - \\
\hline & CD80 & $\uparrow$ \\
\hline & CD86 & $\downarrow$ \\
\hline \multirow{11}{*}{$\begin{array}{l}\text { N2 like } \\
\text { genes }\end{array}$} & Arg1 & $\downarrow$ \\
\hline & $\mathrm{Ccl} 2$ & $\uparrow$ \\
\hline & Ccl5 & - \\
\hline & II-10 & $\uparrow$ \\
\hline & Vegf & $\uparrow$ \\
\hline & Cxcr4 & $\uparrow$ \\
\hline & Mmp-9 & $\downarrow$ \\
\hline & C-myc & - \\
\hline & Stat3 & - \\
\hline & Ccl17 & $\uparrow$ \\
\hline & II-6 & - \\
\hline
\end{tabular}

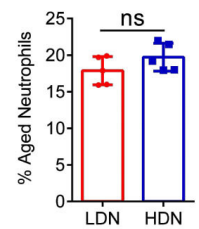

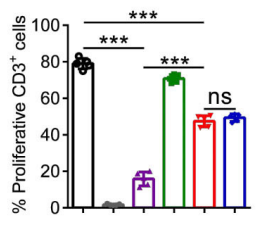

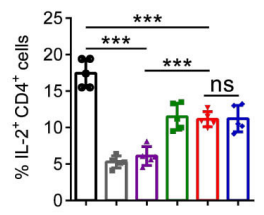

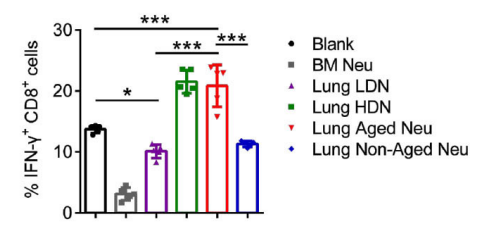

Figure 3 Naged are a unique subset of neutrophils. (A) Principal component analysis (PCA) (left panel) and Volcano Plot (right panel) of RNAseq of the tumor-associated aged neutrophils, tumor-associated non-aged neutrophils and inflammatoryassociated aged neutrophils. Each dot of Volcano Plot representing a gene and genes significantly upregulated in red and downregulated in blue. (B) Heat map of Normalized Enrichment Scores (NES) for selected pathways in tumor-associated aged and non-aged neutrophils. Red, upregulated; blue, downregulated. (C) Analysis of changes in the expression of N1-like and N2-like genome mRNAs of tumor-associated aged neutrophils compared with non-aged neutrophils. (D) Percentage of aged neutrophils among lung HDNs (high-density neutrophils) and LDNs (low-density neutrophils). (E) Percentage of proliferating CFSE-labeled $\mathrm{CD}^{+} \mathrm{T}$ cells when co-cultured with lung HDNs, LDNs, lung aged or non-aged neutrophils from 2 weeks tumorbearing mice. Bone marrow (BM)-derived neutrophils from tumor-bearing mice served as a positive control. (F, G) Flow cytometry analysis of IL-2 production by $\mathrm{CD} 4^{+} \mathrm{T}$ cells $(\mathrm{F})$ and IFN- $\gamma$ production by $\mathrm{CD} 8^{+} \mathrm{T}$ cells $(\mathrm{G})$ when cocultured with lung LDNs, HDNs, aged or non-aged neutrophils and BM-derived neutrophils. Data are presented as the means \pm SD from one representative experiment. Similar results were obtained from three independent experiments, unless indicated otherwise. Statistical analysis was performed by two-tailed unpaired Student's t-test (D) and one-way ANOVA (E, F, G). ${ }^{\star} p<0.05,{ }^{* \star *} p<0.001$. ANOVA, analysis of variance; CFSE, carboxyfluorescein succinimidyl ester; IFN- $\gamma$, interferon $\gamma$; IL-2, interleukin 2; ns, not significant; ROS, reactive oxygen species. 
A

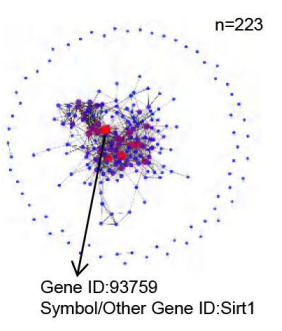

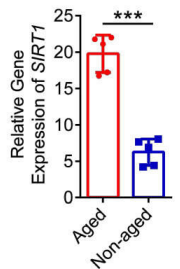
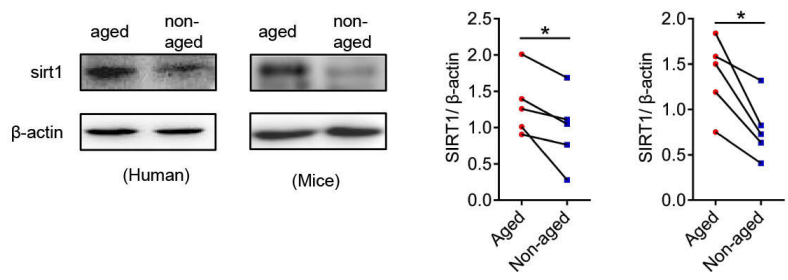

E

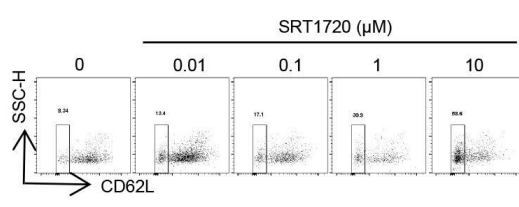

(Human)

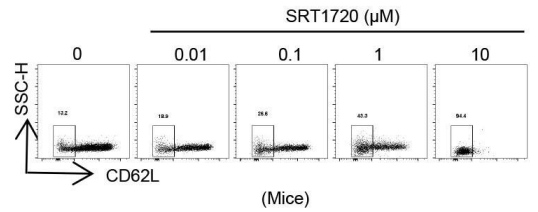

(Mice)
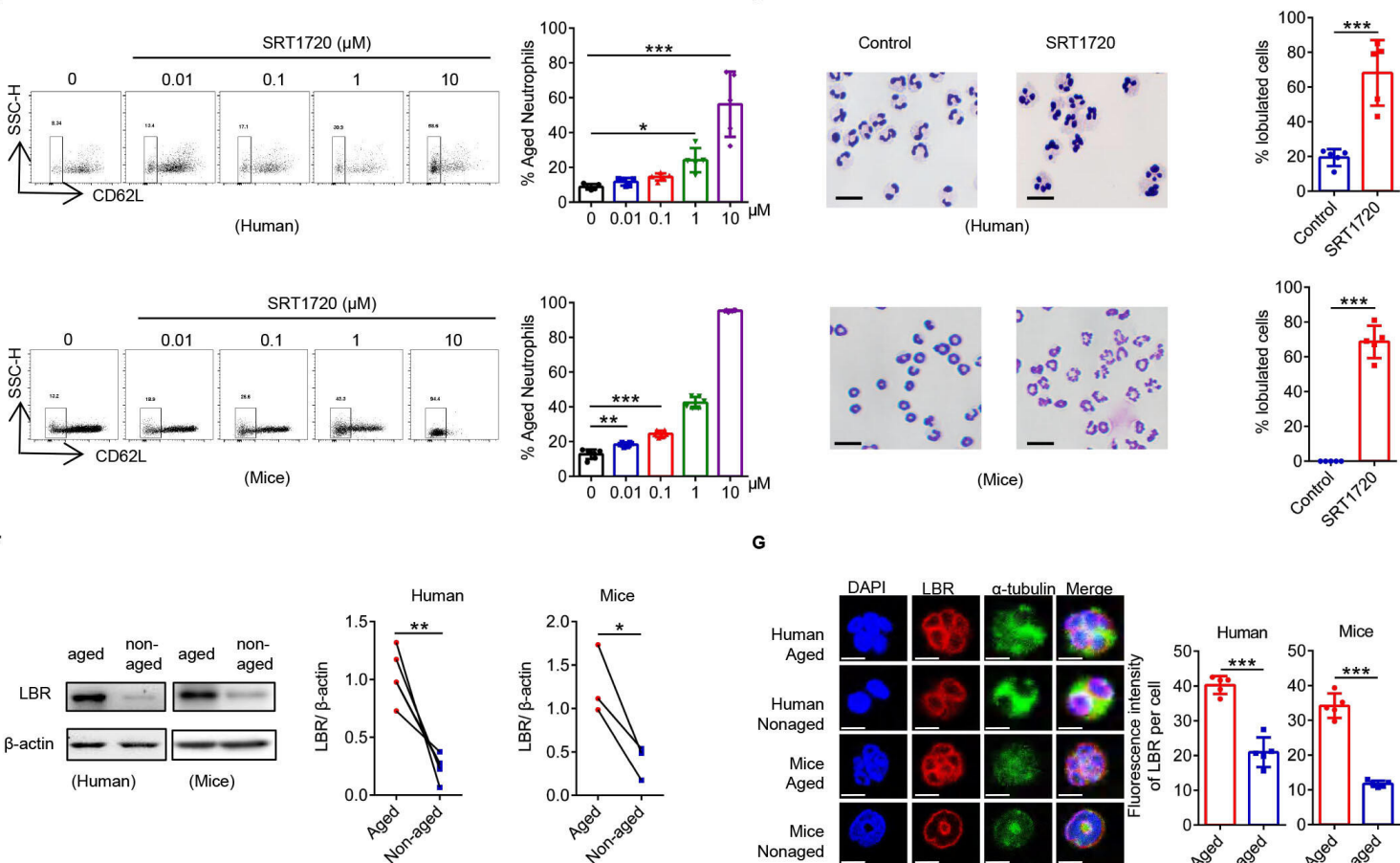

G

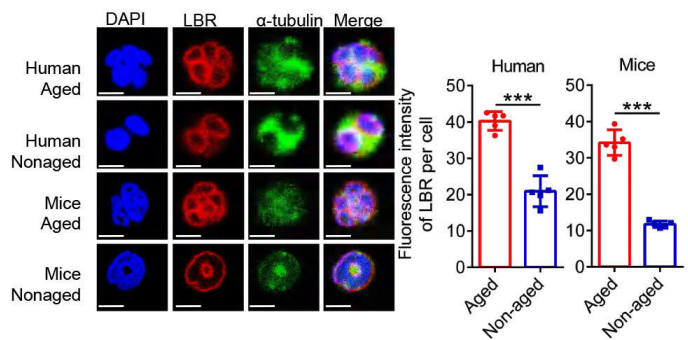

H
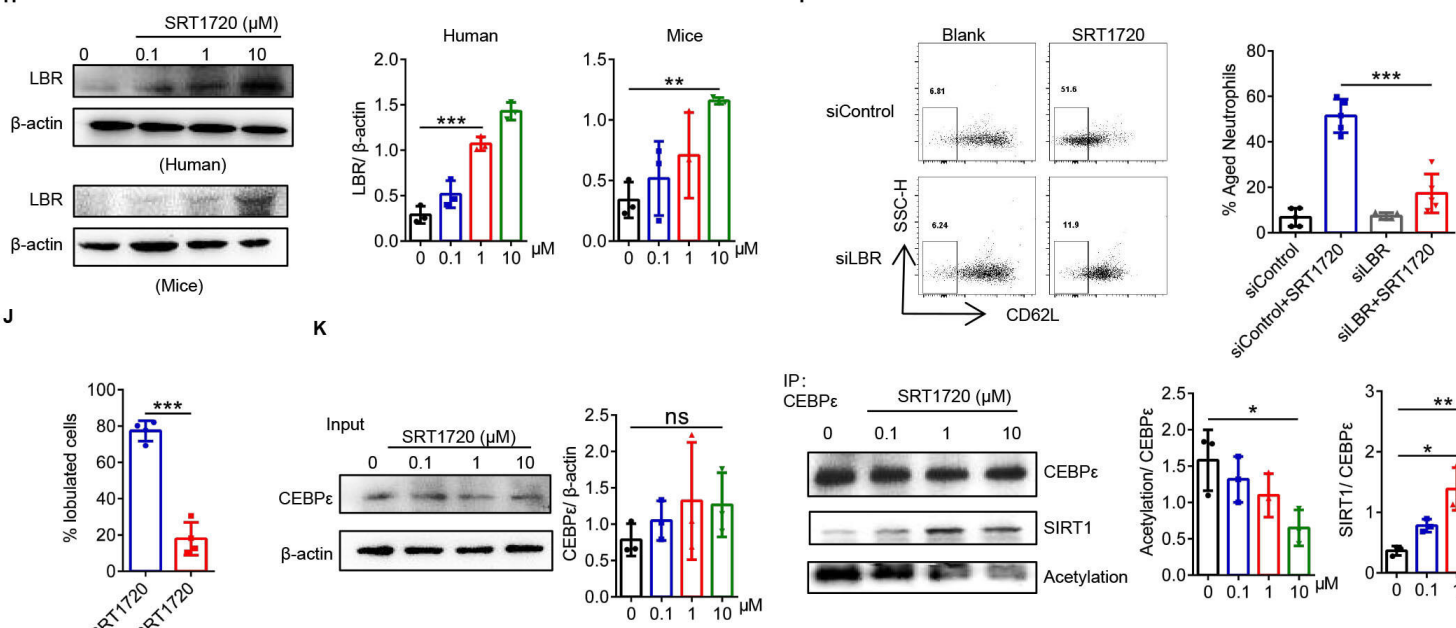

IP:
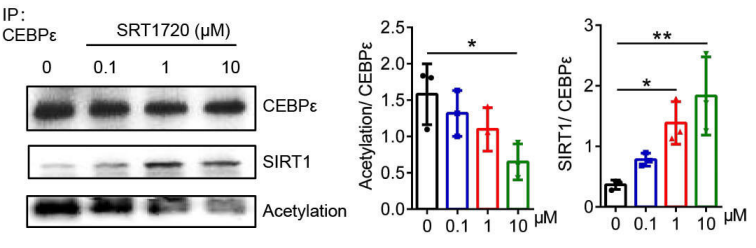

M
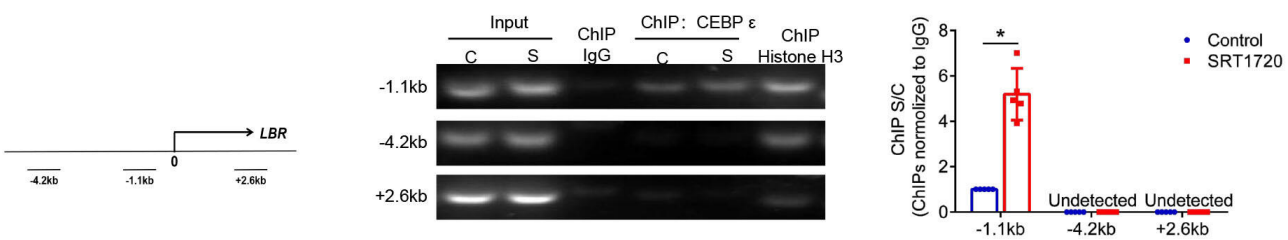

Figure 4 (Continued) 
Figure 4 The SIRT1-C/EBPE-Lamin B receptor (LBR) axis regulates the oversegmentation of Naged. (A) Protein network analysis of RNAseq of differentially expressed transcription factors in tumor-associated aged and non-aged neutrophils. (B) Analysis of the mRNA expression of SIRT1 in lung aged and non-aged neutrophils from 2 weeks tumor-bearing mice. (C) Levels of the SIRT1 protein in aged and non-aged neutrophils in the PB from patients with breast cancer and lung from 2 weeks tumor-bearing mice. (D) Flow cytometry analysis (left panel) and quantification (right panel) of aged neutrophils in the PB from patients with breast fibroadenoma (upper panel) and BM from naïve mice (lower panel) treated with different concentrations of SRT1720 for 4 hours in vitro. (E) Representative images and quantification of Giemsa staining showing the nucleus morphology of neutrophils in PB from patients with breast fibroadenoma (upper panel) and BM from naîve mice (lower panel) treated with SRT1720 for 4 hours in vitro. Scale bar, $5 \mu \mathrm{m}$. (F) levels of the LBR protein in aged and non-aged neutrophils in the PB from patients with breast cancer and lung of 2 weeks tumor-bearing mice. (G) Cellular immunofluorescence staining of aged and nonaged neutrophils from the PB of patients with breast cancer and lung of 2 weeks tumor-bearing mice. Blue, DAPI. Red, LBR. Green, $\alpha$-tubulin. Scale bar, $2 \mu \mathrm{m}$. (H) Levels of the LBR protein on neutrophils in PB from patients with breast fibroadenoma and BM of naïve mice treated with SRT1720 for 4 hours in vitro. (I, J) Flow cytometry analysis (I) and Giemsa staining of the nucleus morphology $(\mathrm{J})$ of siControl-transfected and siLBR-transfected neutrophils derived from the BM of naive mice treated with SRT1720 for 4 hours in vitro. Scale bar, $5 \mu \mathrm{m}$. (K) Co-immunoprecipitation analysis of the acetylation of C/EBPE in neutrophils from the BM of naïve mice treated with SRT1720 for 4 hours in vitro. (L) Three primer sets in the LBR locus used to amplify chromatin immunoprecipitation (ChIP)'d DNA. (M) Distribution of C/EBPE at the LBR locus. C, Control. S, SRT1720. ChIP assays were performed using C/EBPE. ChIP'd DNA was analyzed using qPCR. The bar graph shows the ratios of ChIP'd DNA signals (normalized to lgG). Data are presented as the means $\pm S D$ from one representative experiment. Similar results were obtained from three independent experiments, unless indicated otherwise. Statistical analysis was performed by two-tailed unpaired Student's t-test (B, E, G, J, M), paired Student's t-test (C, F) and one-way ANOVA (D, H, I, K). ${ }^{*} \mathrm{P}<0.05$, ${ }^{* *} \mathrm{p}<0.01$, and ${ }^{* \star *} \mathrm{p}<0.001$. ANOVA, analysis of variance; BM, bone marrow; ns, not significant; PB, peripheral blood.

that compared with tissues from breast fibroadenoma, tissues from breast cancer expressed NAMPT at high levels (figure $7 \mathrm{G}$ and online supplemental figure $7 \mathrm{G}, \mathrm{H}$ ), after excluding the influence of patients' age (online supplemental figure 7I); these cells were predominantly located in the cancer nest rather than the adjacent normal tissues (online supplemental figure 7J). More importantly, the highest NAMPT expression was observed in TNBC (online supplemental figure $7 \mathrm{~K}$ ), and similar results were also obtained from patient serum (figure $7 \mathrm{H}$ and online supplemental figure $7 \mathrm{~L}$ ). NAMPT is an upstream protein of SIRT1, and recombinant NAMPT effectively induced SIRT1 expression in neutrophils (online supplemental figure $7 \mathrm{M}$ ) and promoted their polarization to Naged, changes that were reversed by inhibitors of SIRT1, such as EX527 (figure 7I and J and online supplemental figure $7 \mathrm{~N})$. NAMPT has recently been reported to be secreted by tumor cells and regulated by stress. ${ }^{48}$ Tumor cells from either mice or humans secreted NAMPT in the absence of serum (figure $7 \mathrm{~K}$ and online supplemental figure $7 \mathrm{O}, \mathrm{P})$. Serum-free tumor cell culture supernatants (TCCS) rather than serum-containing TCCS induced the formation of Naged, and this effect was inhibited by the NAMPT inhibitor FK866 and the SIRT1 inhibitor EX527 (figure 7L and online supplemental figure 7Q). In addition, although serum-free TCCS contained less cfDNA than serum-containing TCCS (online supplemental figure 7R), more cfDNA was detected after culturing the neutrophils under serum-free conditions (figure $7 \mathrm{M}$ ).

Therefore, inhibitors of different stages of the pathway were administered to tumor-bearing mice, including FK866 to inhibit NAMPT, vitamin $\mathrm{B}_{3}$ to inhibit SIRT1, Mdivi-1 to inhibit mitophagy, TRO19622 to inhibit the open of mPTP channel, and DNase I to clear the formed
NETs. We did not observe a significant difference in the tumor burden and splenic hyperactivity in the second week of intervention (online supplemental figure 8A). However, only the mice in the FK866 group exhibited significant weight loss. Further examinations revealed that these drugs had no effect on the BM mobilization of neutrophils, while Mdivi-1 reduced the number of neutrophils in the PB and lung, and FK866 produced the opposite effects (online supplemental figure $8 \mathrm{~B}$ ). More importantly, FK866, vitamin B $_{3}$ and Mdivi-1 reduced Naged accumulation in the lung to different degrees (figure $7 \mathrm{~N}$ and online supplemental figure 8C). When neutrophils were sorted from the lung tissue of mice in each group, their abilities to form NETs were reduced in all groups, except the DNaseI group (online supplemental figure 8D), which formed NETs that were cleared in vitro after treatment with supplemental DNaseI (online supplemental figure 8E). After long-term intervention, tumor metastasis was reduced to different degrees (figure 7O). Considering the effect of FK866 on body weight, 4T1-shNAMPT tumor cells were established to determine the mechanism by which tumor-secreted NAMPT regulated Naged and lung metastasis (online supplemental figure $8 \mathrm{~F})$. Reduced NAMPT expression was verified in tumors in vivo (online supplemental figure 8G), but no significant difference in the tumor burden was observed in the early stage (online supplemental figure $8 \mathrm{H}$ ). We also detected decreased mobilization of neutrophils in the $\mathrm{BM}$ and increased numbers of neutrophils in the lungs of the shNAMPT group (online online supplemental figure 8I). More importantly, the Naged population was significantly reduced in the lung (figure 7P), and tumor metastasis was also significantly reduced (figure $7 \mathrm{Q}$ ). Based on these results, tumor-secreted NAMPT may be a key factor 
A

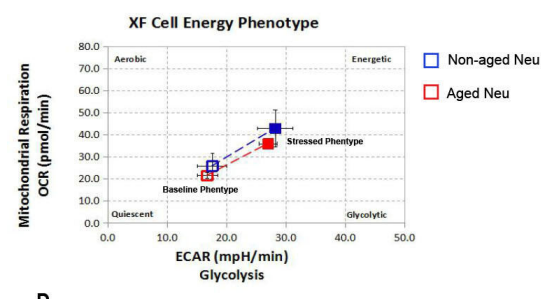

B

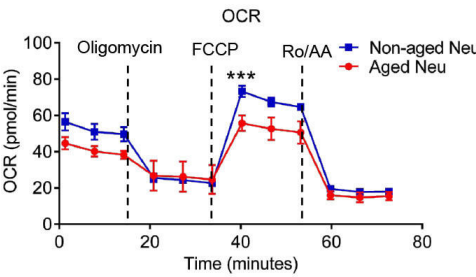

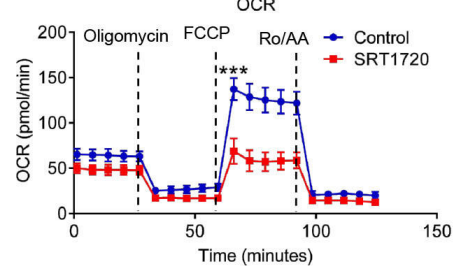

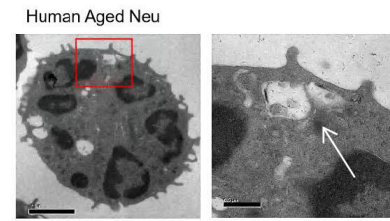

Human Non-Aged neu
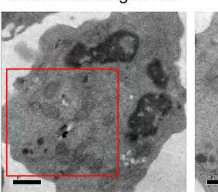
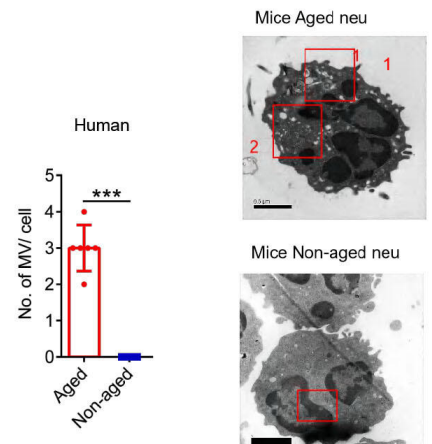

Mice Non-aged neu
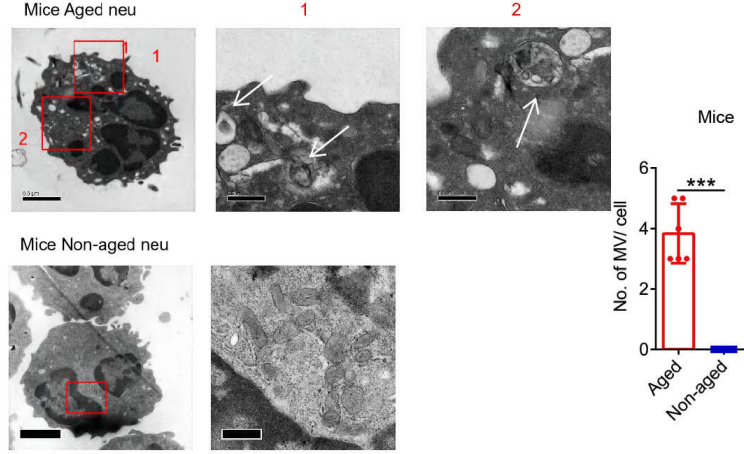

E
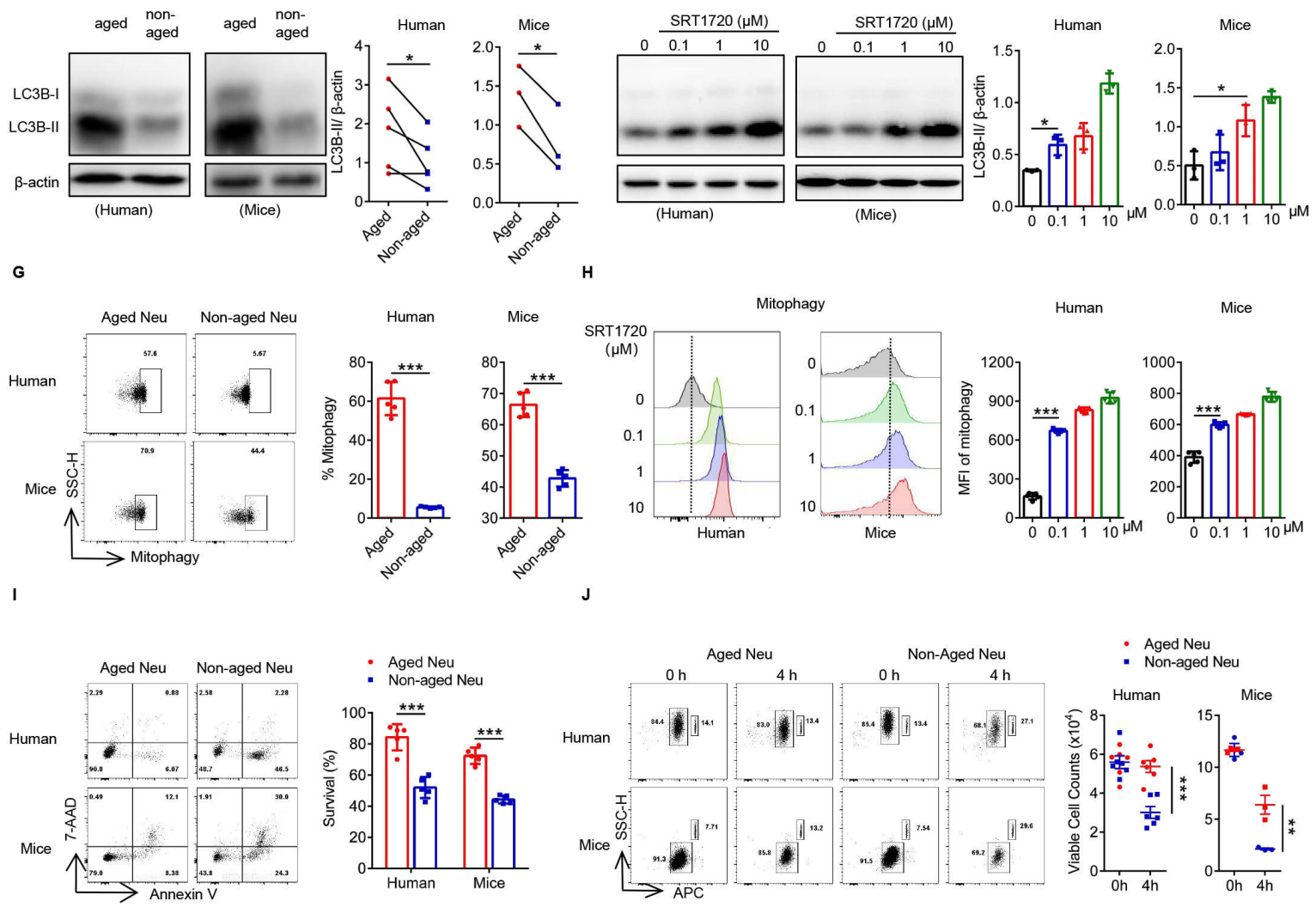

K
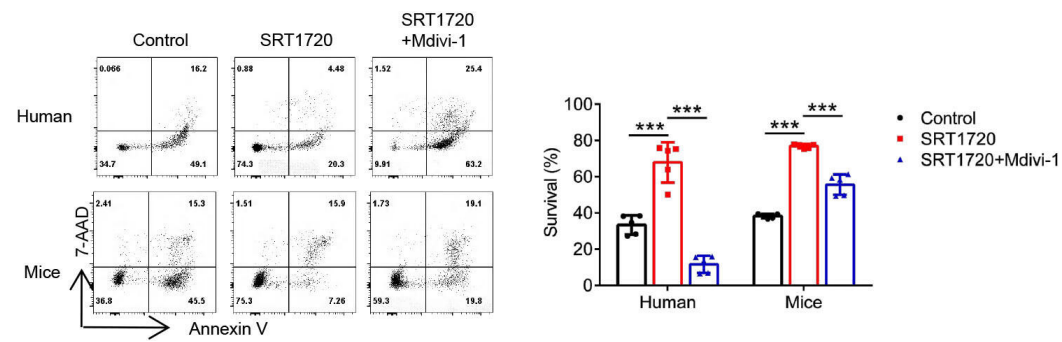

Figure 5 (Continued) 
Figure 5 SIRT1 prolongs the survival of Naged by inducing mitophagy. (A) Analysis of the cellular energy phenotype of aged and non-aged neutrophils from the lungs of 2 weeks tumor-bearing mice. (B) The oxygen consumption rate (OCR) of aged and non-aged neutrophils from 2 weeks tumor-bearing mice was measured under basal conditions and in response to treatment with the indicated drugs ( $1 \mu \mathrm{M}$ Oligomycin, $1 \mu \mathrm{M}$ FCCP, and $2.5 \mu \mathrm{M}$ Rotenone and Antimycin A). (C) The OCR of neutrophils from the BM of naïve mice treated with SRT1720 was measured under basal conditions and in response to treatment with the indicated drugs ( $1 \mu \mathrm{M}$ Oligomycin, $1 \mu \mathrm{M}$ FCCP, and $2.5 \mu \mathrm{M}$ Rotenone and Antimycin A). (D) Representative TEM images and quantification of the microvesicles (MV) of aged and non-aged neutrophils in PB from patients with breast cancer (left panel) and lung of 2 weeks tumor-bearing mice (right panel). Scale bar, $1 \mu \mathrm{m}$ (left panel), $0.5 \mu \mathrm{m}$ (right panel). The white arrow, MV. (E) Levels of the LC3B protein in aged and non-aged neutrophils in the PB from patients with breast cancer and lung of 2 weeks tumor-bearing mice. $(\mathrm{F})$ Levels of the LC3B protein on neutrophils in the PB from patients with breast fibroadenoma and the BM of naïve mice treated with SRT1720 for 4 hours in vitro. (G) Flow cytometry analysis of mitophagy in neutrophils from the PB of patients with breast cancer and lung of 2 weeks tumor-bearing mice, in condition with pepstatin $A$ and leupeptin (inhibitors of autophagy clearance) for 4 hours in vitro. (H) Flow cytometry analysis of mitophagy in neutrophils from the PB of patients with breast fibroadenoma (left panel) and the BM of naïve mice (right panel) treated with SRT1720, in condition with pepstatin A and leupeptin (inhibitors of autophagy clearance) for 4 hours in vitro. (I) Flow cytometry analysis of the survival of aged and non-aged neutrophils from the PB of patients with breast cancer and lung of 2 weeks tumor-bearing mice for 4 hours in vitro. $(\mathrm{J})$ Flow cytometry analysis of the absolute living cells count of aged and non-aged neutrophils from the PB of patients with breast cancer and lung of 2 weeks tumor-bearing mice for 4 hours in vitro. APC labeled CD66b of human samples and Ly-6G of mice samples, neutrophils were presented at the left gate and counting beads at the right). (K) Flow cytometry analysis of the survival of neutrophils from the PB of patients with breast fibroadenoma and the BM of naïve mice treated with SRT1720 in the presence or absence of Mvidi- 1 for 4 hours in vitro. Data are presented as the means \pm SD from one representative experiment. Similar results were obtained from three independent experiments, unless indicated otherwise. Statistical analysis was performed by repeated-measures ANOVA (B, C), two-tailed unpaired Student's $t$ test (D, G, I), paired Student's t test (E), one-way ANOVA $(\mathrm{F}, \mathrm{H}, \mathrm{K})$ and two-way ANOVA $(\mathrm{J}) .{ }^{*} \mathrm{P}<0.05,{ }^{* *} \mathrm{p}<0.01$, and ${ }^{* * *} \mathrm{p}<0.001$. ANOVA, analysis of variance; APC, allophycocyanin; $\mathrm{BM}$, bone marrow; FCCP, carbonyl cyanide p-trifluoromethoxy-phenylhydrazone; MFI, mean fluorescence intensity; ns, not significant; PB, peripheral blood; TEM, transmission electron microscopy.

that activates SIRT1 to polarize neutrophils to Naged and form mitochondria-dependent NETs to promote the lung metastasis of breast cancer.

\section{DISCUSSION}

The expression level of CD62L on the surface divides neutrophils into three subsets: CD62L ${ }^{\text {low }}, \mathrm{CD} 62 \mathrm{~L}^{\text {mid }}$ and CD62L ${ }^{\text {hi }}$ neutrophils, which performs different functions. Aged neutrophils were commonly defined as $\mathrm{CXCR} 4{ }^{\text {hi }} \mathrm{C}$ D62 $\mathrm{L}^{\text {low }}$ and non-aged neutrophils were $\mathrm{CXCR} 4^{+} \mathrm{CD} 62 \mathrm{~L}^{\text {hi }}$

${ }^{39}$. Previous studies have suggested that the expression of surface CXCR4 by neutrophils is an important cause of its chemotaxis to lung tissue. CD62L, as an adhesion molecule, plays a role in determining activation state, ${ }^{49}$ and has been found to help defining the maturation state of neutrophils. ${ }^{39}$ Therefore, our study pays more attention to whether CD62L on neutrophils can be used to differentiate the senility of neutrophils.

Neutrophils mature and exit from the BM, then age and subsequently are cleared by local macrophages or return to the BM to maintain myeloid cell stability. According to previous studies, aged neutrophils display impaired migration, decreased proinflammatory effects, and are waiting to be cleared.$^{50}$ Subsequently, Uhl $e t a \tilde{l}^{51}$ found that aged neutrophils moved faster and were the first responders to inflammation. Zhang et a $\tilde{p}^{2}$ further confirmed that the proinflammatory activity of neutrophils positively correlated with their aging in the circulation. Subsequent studies illustrated that the persistence of aged neutrophils was responsible for lingering inflammation in individuals with pulmonary fibrosis. ${ }^{53}$ However, the role of aged neutrophils in tumors was unclear. We first documented that tumor-associated aged neutrophils (Naged) significantly accumulated in the lung tissue during the niche formation period. More importantly, the transcriptomic characteristics of these Naged neutrophils were completely different from tumor-associated non-aged neutrophils or inflammation-induced aged neutrophils. Furthermore, as predicted by the sequencing results, we found the survival of Naged was prolonged due to proapoptotic genes downregulation and antiapoptotic genes upregulation. The expression of adhesion-related proteins was increased in Naged. Notably, the capacities of angiogenesis and immunosuppressive cells recruitment were significantly increased, and the levels of chemokines and inflammatory responses were altered, indicating that Naged are an important participant in tumor metastasis.

Reminiscent of the classification of M1/M2 macrophages, neutrophils are also classified into N1 and N2 types. ${ }^{15}$ Another classic model for studying neutrophil heterogeneity is the LDN and HDN dichotomy. ${ }^{16}$ LDNs are more similar to PMN-MDSCs because of their immunosuppressive function, while HDNs are superior at killing tumor cells. As shown in our study, Naged did not belong to the N1 or N2 types, based on a comparison of known characteristic N1/N2 genes. In addition, Naged were not typical PMN-MDSCs, and their inhibitory effects on T cell proliferation or the secretion of IFN- $\gamma$ and IL-2 were not as prominent as LDNs. More importantly, the proportion of Naged was equivalent in LDNs and HDNs. A further analysis of the differences in expression of transcription 

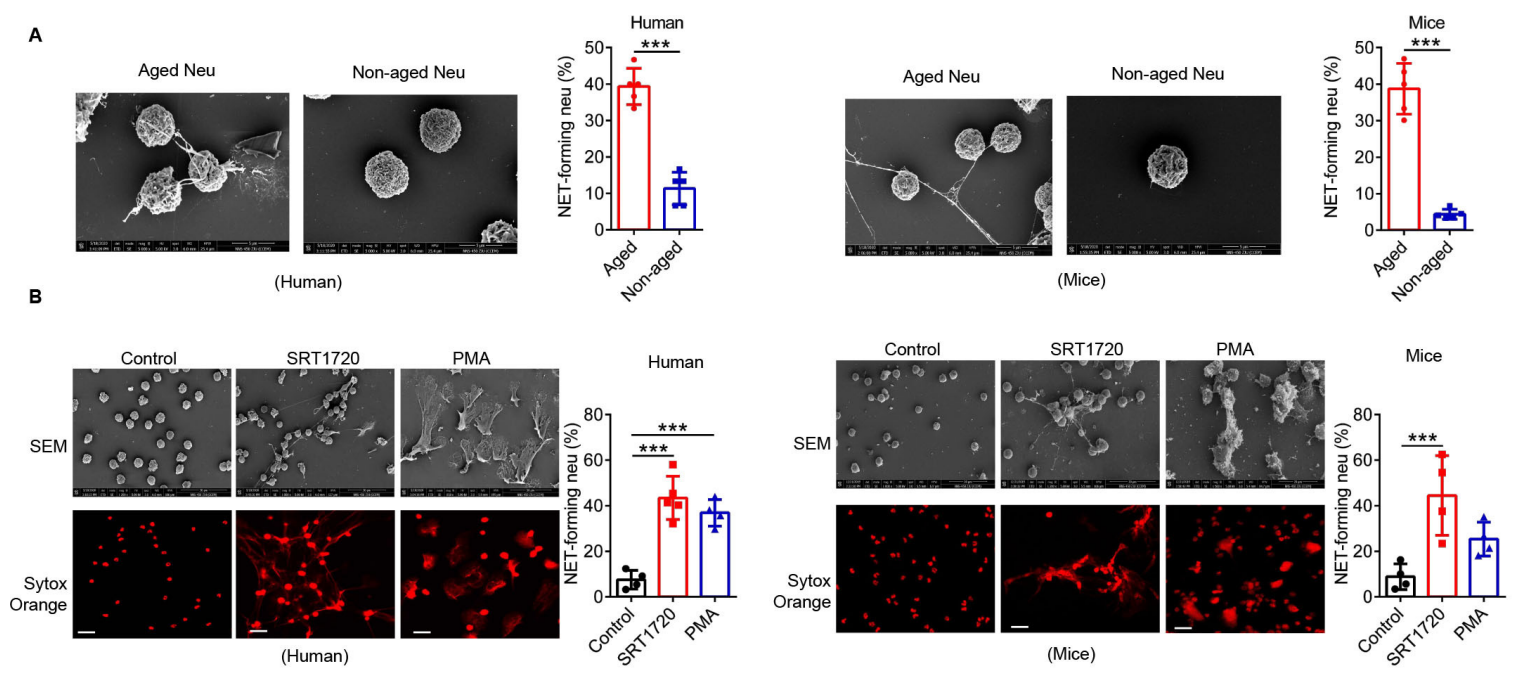

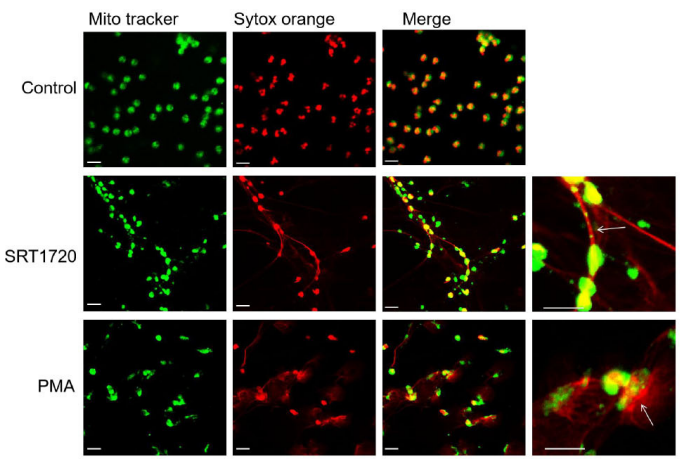

(Human)

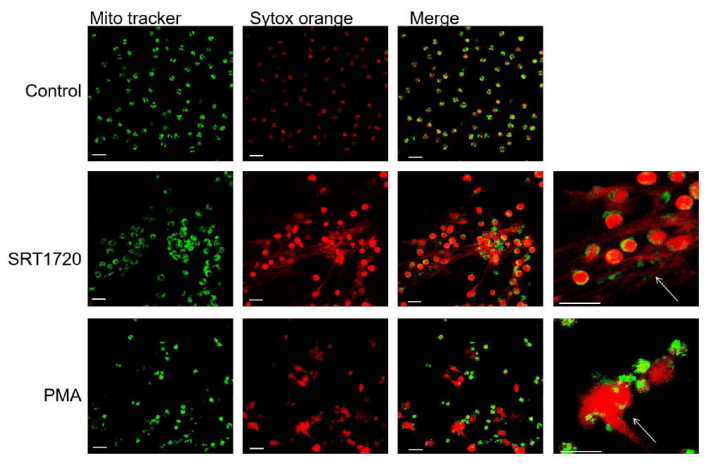

(Mice)

D

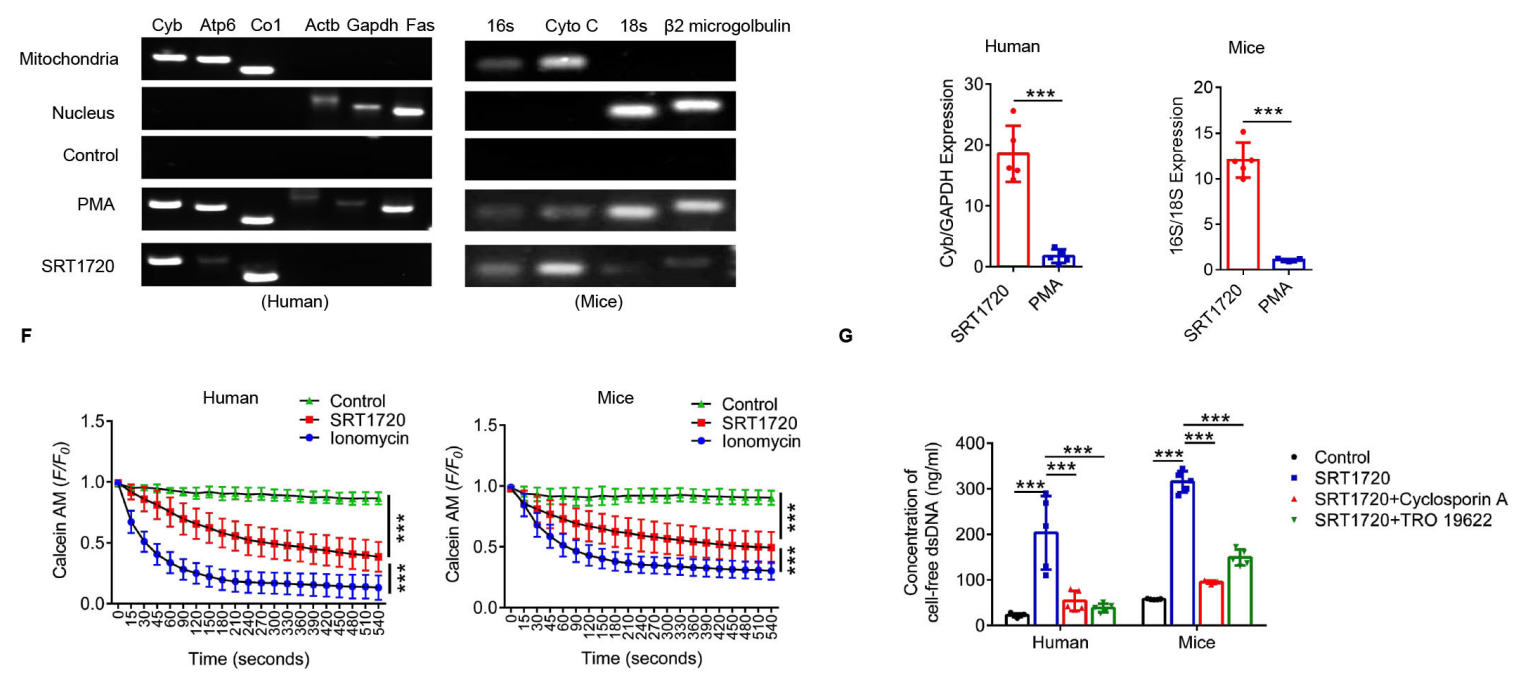

H
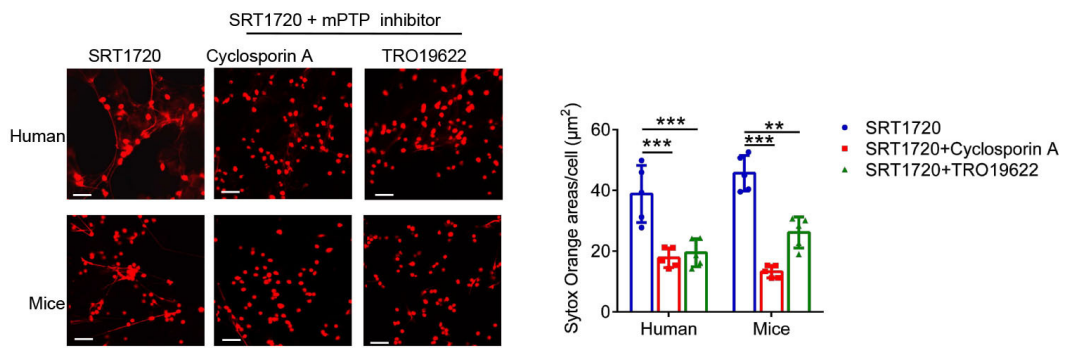

Figure 6 (Continued) 
Figure 6 SIRT1-induced Naged form mitochondria-dependent vital NETs via opening mPTP channels. (A) Scanning electron microscopy (SEM) images and quantification of extracellular traps (NETs) produced by aged and non-aged neutrophils from the PB of patients with breast cancer and lung of 2 weeks tumor-bearing mice. (B) Images of SEM (upper panel) and immunofluorescence staining (lower panel) of NETs produced by neutrophils from the PB of patients with breast fibroadenoma and the BM of naïve mice treated with SRT1720 and PMA, respectively. Red, Sytox Orange. Scale bar, 10 $\mu$ m. (C) Cellular immunofluorescence staining NET components produced by neutrophils from the PB of patients with breast fibroadenoma and the BM of naïve mice treated with SRT1720 and PMA, respectively. Green, Mito Tracker. Red, Sytox Orange. Scale bar, $10 \mu \mathrm{m}$. (D, E) PCR analysis of NET components produced by neutrophils from the PB of patients with breast fibroadenoma and the BM of naïve mice treated with SRT1720 and PMA. Mitochondrial and nuclear DNA isolated from purified neutrophils was used as controls. Mitochondrial and nuclear genes were amplified from different DNA templates. (F) Opening of the mPTP in neutrophils from the PB of patients with breast fibroadenoma and the BM of naïve mice treated with SRT1720. Ionomycin was used as a positive control. (G) Concentration of cell-free DNA (cfDNA) in cultured supernatants from neutrophils in the PB of patients with breast fibroadenoma and the BM of naïve mice treated with SRT1720 in the presence or absence of TRO19622 or Ciclosporin A (mPTP inhibitors). (H) Immunofluorescence staining (left panel) and quantification (right panel) for NETs in neutrophils from the PB of patients with breast fibroadenoma and BM of naïve mice treated with SRT1720 in the presence or absence of TRO19622 or Ciclosporin A (mPTP inhibitors). Scale bar, $10 \mu \mathrm{m}$. Data are presented as the means \pm SD from one representative experiment. Similar results were obtained from three independent experiments, unless indicated otherwise. Statistical analysis was performed by two-tailed unpaired Student's $t$ test $(A, E)$, one-way ANOVA $(B, G, H)$ and repeated-measures ANOVA (F). ${ }^{* *} \mathrm{p}<0.01,{ }^{* \star *} \mathrm{p}<0.001$. ANOVA, analysis of variance; BM, bone marrow; mPTP, mitochondrial permeability transition pore; NETs, neutrophil extracellular traps; ns, not significant; PB, peripheral blood.

factors related to MDSC function between Naged and LDNs/HDNs revealed that LDNs expressed more MDSCrelated transcription factors, while Naged did not, particularly factors associated with typical characteristics such as Arginase 1, reactive oxygen species, and reactive nitrogen species. Despite these results, it remains unclear whether these different populations represent bona fide subsets or simply activation/polarization states. Thus, we identify potential gaps in our knowledge that may further advance our current understanding of neutrophils heterogeneity by single cell sequencing of neutrophils.

The lobulated state of neutrophils are considered a sign of maturity. ${ }^{54}$ Researchers had worked to promote the maturation of neutrophils, particularly in leukemia. We found that the core transcription factor SIRT1 regulated the lobulated state of tumor-associated neutrophils. Interestingly, the Lamin protein and the Lamin receptor on the nuclear membrane regulated nuclear compartmentalization, and high expression of LBR promoted the formation of nuclear membrane wrinkles and nuclear oversegmentation. ${ }^{55}$ We performed electroporation in vitro to knock out $L B R$ in neutrophils and further demonstrated the SRT1720-induced neutrophil oversegmentation was mediated by LBR. Unfortunately, ideal data from human neutrophils were unable to be obtained due to the low tolerance of human neutrophils to electroporation. Furthermore, SIRT1 functioned as a deacetylase for $\mathrm{C} / \mathrm{EBP} \varepsilon$, enabling it to bind the $L B R$-specific promoter locus to promote $L B R$ transcription.

SIRT1 was considered a multifunctional transcription factor. ${ }^{56}$ In addition to its role as a deacetylase, it also regulates various types of energy metabolism. Neutrophils had long been regarded as cells that lack mitochondria, and the production of their energy sources depended on the glycolysis and pentose phosphate pathways. ${ }^{57}$ However, accumulating evidences indicate that mitochondria in neutrophils were not as rare as reported and their OCR level was not low either, but we found that the OCR of Naged was limited comparing with non-naged. Further exploration revealed that SIRT1 regulated mitophagy in Naged. Mitophagy reduced the mitochondrial content and OXPHOS of neutrophils and, more importantly, avoided mitochondria-dependent caspase apoptosis, consistent with the sequencing results showing that the antiapoptotic pathway was upregulated and the proapoptotic pathway was downregulated in the Naged group. Naged maintained their lifespan through mitophagy, which appeared is a key factor underlying their increased abundance in lung tissue.

The role of NETs in promoting tumor progression has received increasing attention in recent years. ${ }^{21} 2258$ NETs enhanced the migration and invasion capacity of tumor cells. ${ }^{59}$ More interestingly, NETs awakened dormant tumor cells by lysing the extracellular matrix, resulting in tumor recurrence and metastasis. ${ }^{20}$ NETs formed by neutrophils have been shown to promote the liver metastasis of breast cancer through DNA receptors on the surface of the cell membrane. ${ }^{58}$ Using cellular immunofluorescence staining and electron microscopy, we showed that Naged had stronger ability to form NETs than non-aged neutrophils. Further video and in vitro studies showed that the Naged-generated NETs captured tumor cells. Tissue immunofluorescence staining confirmed that the NETs produced by Naged were spatially related to tumor cells. Importantly, NETs formed by Naged did not affect tumor cell proliferation or kill tumor cells, but clearing NETs with DNaseI definitely reduced lung metastasis. Taken together, Naged may accelerate lung metastasis by trapping tumor cells through increased NET formation. Although we cannot yet specific remove aged neutrophils, we can see a significant reduction in lung metastasis after the early depletion of total neutrophils, which means the 
A

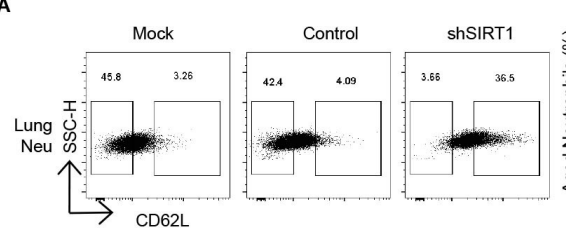

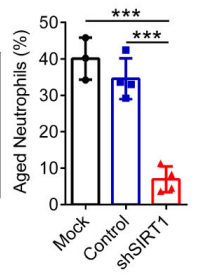

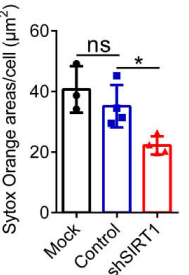

B
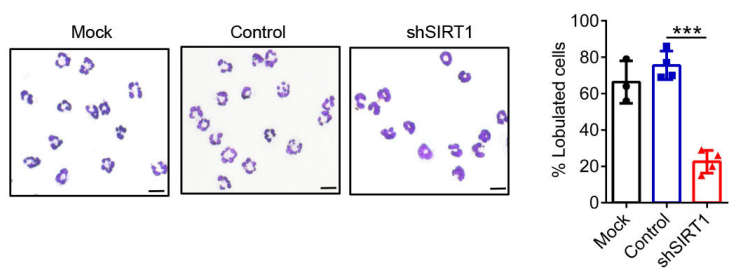

D
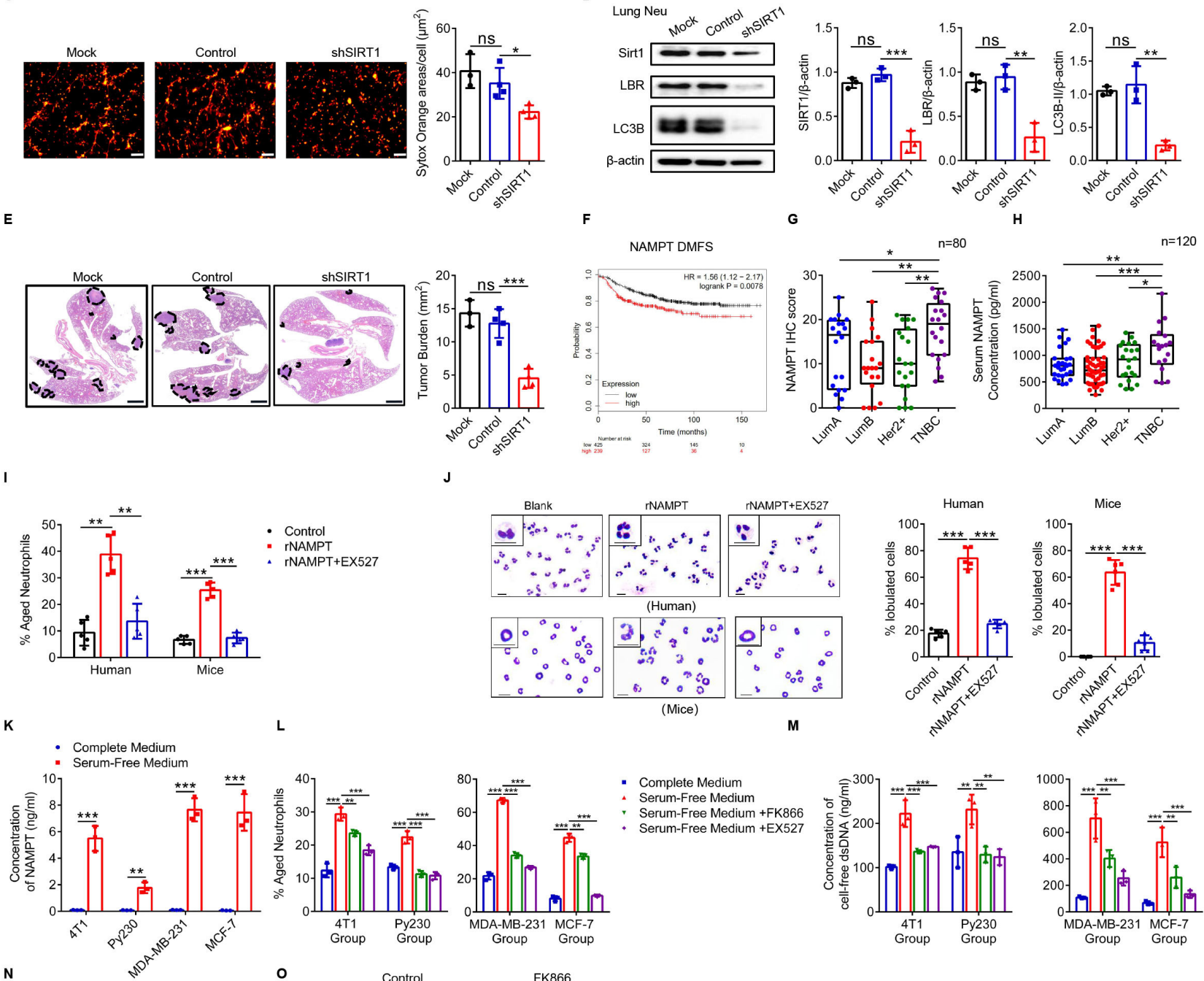

o
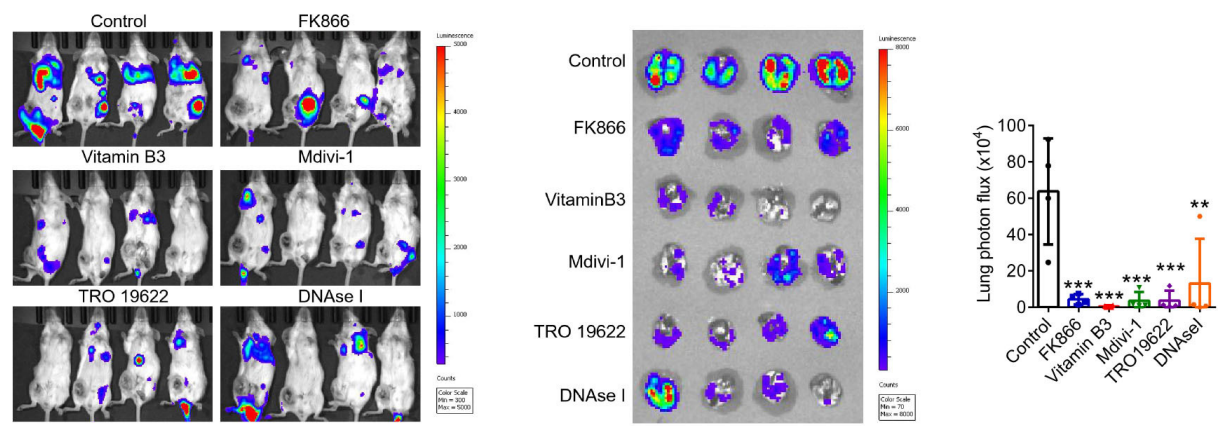

Figure 7 (Continued)
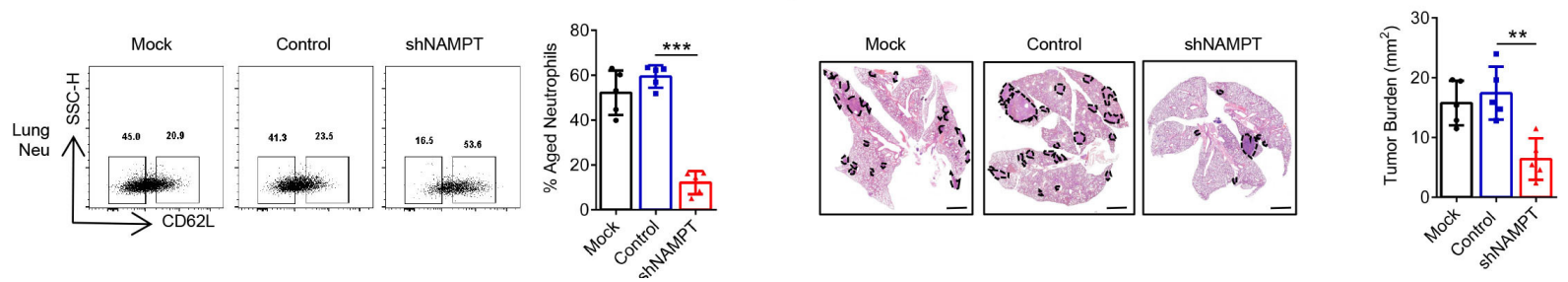
Figure 7 Tumor-secreted NAMPT is responsible for the expression of SIRT1 on Naged, promoting premetastatic niche formation. (A) Flow cytometry analysis (left panel) and quantification (right panel) of lung-infiltrating aged neutrophils from 2 weeks tumor-bearing mice with bone marrow (BM) reconstruction. (B) Representative nucleus morphology of neutrophils in lung from 2 weeks tumor-bearing mice with BM reconstruction. Scale bar, $5 \mu \mathrm{m}$. (C) Immunofluorescence staining and quantification of NETs generated by neutrophils in the lungs of 2 weeks tumor-bearing mice with BM reconstruction. Red, Sytox Orange. Scale bar, $50 \mu \mathrm{m}$. (D) Levels of the SIRT1, LBR and LC3B protein in the neutrophils from lung of 2 weeks tumor-bearing mice with BM reconstruction. (E) Images of H\&E staining in lung sections (left panel) and quantification (right panel) of lung metastasis in 6 week tumor-bearing mice with BM reconstruction. Scale bar, $1 \mathrm{~mm}$. (F) Analysis of the correlations between NAMPT expression in primary tumors with distant metastasis-free survival (DMFS) in patients with breast cancer from the TCGA database. (G) Quantification of NAMPT Immunohistochemical staining in patients with different molecular subtypes of breast cancer. (H) ELISA of serum NAMPT levels in patients with different molecular subtypes of breast cancer. (I, J) Percentage of aged neutrophils (I) and representative nucleus morphology $(\mathrm{J})$ of neutrophils in PB from patients with breast fibroadenoma and the BM from naïve mice treated with rNAMPT in the presence or absence of the EX527 (SIRT1 inhibitor). Scale bar, $5 \mu \mathrm{m}$. (K) ELISA of the NAMPT concentrations in different conditioned tumor cell cultured supernatants (TCCS). (L, M) Flow analysis of aged neutrophils (L) and cfDNA (M) from neutrophils in the PB of patients with breast fibroadenoma and the BM of naïve mice treated with different TCCS in the presence or absence of the FK866 (NAMPT inhibitor) and EX527 (SIRT1 inhibitor). Flow analysis of lung-infiltrating aged neutrophils in tumor-bearing mice after the administration of multiple interventions for 2 weeks. Data were all compared with the control group. (O) Representative bioluminescent images (left panel) and quantification (right panel) in the lungs of BALB/c mice injected with luciferase-expressing 4T1 cells and administered multiple interventions for 2 weeks. (P) Flow cytometry analysis (left panel) and quantification (right panel) of lung-infiltrating aged neutrophils in the second week after animals were inoculated with Mock, Control or sh-NAMPT cell lines. (Q) Images of H\&E staining in lung sections (left panel) and quantification (right panel) of lung metastasis in 6 week tumor-bearing mice. Scale bar, $1 \mathrm{~mm}$. Data are presented as the means $\pm S D$ of one representative experiment. Similar results were obtained from three independent experiments. Statistical analysis was performed by two-tailed unpaired Student's t-test (K) and one-way ANOVA (A-E,I, J, L-Q) and Mann-Whitney $U$ tests $(G, H)$. ${ }^{* *} p<0.01$, and ${ }^{* * *} p<0.001$. ANOVA, analysis of variance; cfDNA, cell-free dsDNA; NETs, neutrophil extracellular traps; ns, not significant; PB, peripheral blood.

early lung recruitment and capture capabilities of aged neutrophils could help tumor metastasis, while the late depletion fails to improve outcomes since tumor cells has already settled in the lung and proliferated.

PMA is a representative molecule that induced NET formation. We made a great effort to compare the morphological and signaling pathways of PMA-induced and SIRT1-induced NETs. More importantly, SIRT1induced NETs were dominated by mitochondrial DNA, with the absence of Cit-Histone H3. We emphasized that the Naged were living cells and formed vital NETs. Thus, Naged might perform other functions in addition to producing NETs, and NETs were likely one of the important mechanisms by which Naged promoted the lung metastasis of breast cancer. This study is the first conducted in the field of cancer to show that vital NETs form from mitochondrial DNA to generate a premetastatic niche that promoted breast cancer progression.

Based on the results described above, we performed a variety of interventions centered on SIRT1-Naged. Vitamin $\mathrm{B}_{3}$ inhibited SIRT1 activation in neutrophils by tumor cells, Mdivi-1 blocked mitophagy and promoted Naged death, TRO19622 prevented DNA release from mitochondrial mPTP channels, and DNaseI removed the formed NETs; all of these agents prevented tumor metastasis to varying degrees but did not exert a significant effect on the primary tumor. Importantly, vitamin $B_{3}$ was an oral drug that was commonly used in clinical practice, and its inhibitory effect on Naged may expand its potential clinical applications. It seems a very promising drug for preventing tumor metastasis. Undoubtedly, conditional knockout mice is needed to further confirm the importance of SIRT1-Naged axis, which is the direction for our future research.

Furthermore, whether the specificity of aged neutrophils in breast cancer lung metastasis can be derived into widespread tumors is a question worthy of further investigation. We found that aged neutrophils accumulate in lung tissue of the B16F10 melanoma but not in LLC lung cancer (data were not show), while both of the two models develop lung metastases. This indicates that aged neutrophils cannot be considered to be the pancause of tumor lung metastasis, further study is needed to figure out whether they can indicate a group of tumors for metastasis.

In summary, breast tumor cells secrete NAMPT to activate SIRT1 in neutrophils, which polarize the neutrophils into Naged. Naged extend their lifespan through mitophagy. Thus, Naged continuously accumulate in the lung and produced vital NETs to capture tumor cells, thereby promoting the lung metastasis of breast cancer. Based on these findings, Naged are a potential target cell type to prevent the lung metastasis of breast cancer.

\section{Author affiliations}

${ }^{1}$ Key Laboratory of Tumor Microenvironment and Immune Therapy of Zhejiang Province, The Second Affiliated Hospital, School of Medicine, Zhejiang University, Hangzhou, Zhejiang, People's Republic of China

${ }^{2}$ Department of Breast Surgery, The Second Affiliated Hospital, School of Medicine, Zhejiang University, Hangzhou, Zhejiang, People's Republic of China

${ }^{3}$ Cancer Center, Zhejiang University, Hangzhou, Zhejiang, People's Republic of China 
${ }^{4}$ Department of Breast Surgery, The First Affiliated Hospital, Wenzhou Medical University, Wenzhou, Zhejiang, People's Republic of China

${ }^{5}$ Department of Oncology, The Second Affiliated Hospital, School of Medicine, Zhejiang University, Hangzhou, Zhejiang, People's Republic of China ${ }^{6}$ Department of Gynecology, The Second Affiliated Hospital, School of Medicine, Zhejiang University, Hangzhou, Zhejiang, People's Republic of China ${ }^{7}$ Department of Surgical Oncology, Zhejiang Taizhou Municipal Hospital, Taizhou, Zhejiang, People's Republic of China

${ }^{8}$ Department of Thoracic Surgery, The Second Affiliated Hospital, School of Medicine, Zhejiang University, Hangzhou, Zhejiang, People's Republic of China ${ }^{9}$ Department of Pathology, The Second Affiliated Hospital, School of Medicine, Zhejiang University, Hangzhou, Zhejiang, People's Republic of China

${ }^{10}$ Department of Medicine and Department of Microbiology and Immunology, James Graham Brown Cancer Center, University of Louisville, Louisville, Kentucky, USA

Correction notice This article has been corrected since it was first published. Affiliations 2, 3 and 4 were listed in the wrong order.

Contributors $\mathrm{CY}, \mathrm{ZW}$ and $\mathrm{JH}$ developed the concept and design of the study. $\mathrm{CY}, \mathrm{ZW}, \mathrm{LL}$ and $\mathrm{KS}$ performed the experiments and analyzed the data. FJ and LZ performed RNA-seq data analysis. JP, ZZ, XJ, SS, HW and FQ collected and processed patient samples. PW, FQ, JY and JH supervised the study. CY, ZW, LL and $\mathrm{JH}$ wrote the manuscript. All authors contributed to the critical review of the manuscript.

Funding This work was supported by grants from the National Natural Science Foundation of China (81930079 and 81872317 to JH, 81902981 to ZW, 81802633 to SS), China Postdoctoral Science Foundation Grant (2019M652115 to SS), Zhejiang Science and Technology Major Program for Research and Development (2018C03020 to JH). National Zhejiang Health Commission Joint Key Program (WKJ-ZJ-1803 to JH).

Competing interests None declared.

Patient consent for publication Not applicable.

Ethics approval The protocols for the use of human and mouse cells and tissues were approved by the Ethics Committee of Second Affiliated Hospital of Zhejiang University School of Medicine in accordance with the Declaration of Helsinki.

Provenance and peer review Not commissioned; externally peer reviewed.

Data availability statement All data relevant to the study are included in the article or uploaded as online supplemental information.

Supplemental material This content has been supplied by the author(s). It has not been vetted by BMJ Publishing Group Limited (BMJ) and may not have been peer-reviewed. Any opinions or recommendations discussed are solely those of the author(s) and are not endorsed by BMJ. BMJ disclaims all liability and responsibility arising from any reliance placed on the content. Where the content includes any translated material, BMJ does not warrant the accuracy and reliability of the translations (including but not limited to local regulations, clinical guidelines, terminology, drug names and drug dosages), and is not responsible for any error and/or omissions arising from translation and adaptation or otherwise.

Open access This is an open access article distributed in accordance with the Creative Commons Attribution Non Commercial (CC BY-NC 4.0) license, which permits others to distribute, remix, adapt, build upon this work non-commercially, and license their derivative works on different terms, provided the original work is properly cited, appropriate credit is given, any changes made indicated, and the use is non-commercial. See http://creativecommons.org/licenses/by-nc/4.0/.

\section{ORCID iD}

Jian Huang http://orcid.org/0000-0002-7474-2950

\section{REFERENCES}

1 Kaplan RN, Riba RD, Zacharoulis S, et al. VEGFR1-positive haematopoietic bone marrow progenitors initiate the pre-metastatic niche. Nature 2005;438:820-7.

2 Liu Y, Cao X. Characteristics and significance of the pre-metastatic niche. Cancer Cell 2016;30:668-81.

3 Peinado $\mathrm{H}$, Zhang H, Matei IR, et al. Pre-metastatic niches: organspecific homes for metastases. Nat Rev Cancer 2017;17:302-17.

4 Hoshino A, Costa-Silva B, Shen T-L, et al. Tumour exosome integrins determine organotropic metastasis. Nature 2015;527:329-35.
5 Tichet M, Prod'Homme V, Fenouille N, et al. Tumour-derived SPARC drives vascular permeability and extravasation through endothelial VCAM1 signalling to promote metastasis. Nat Commun 2015;6:6993.

6 Kersten K, Coffelt SB, Hoogstraat M, et al. Mammary tumorderived CCL2 enhances pro-metastatic systemic inflammation through upregulation of IL $1 \beta$ in tumor-associated macrophages. Oncoimmunology 2017;6:e1334744.

7 Castaño Z, San Juan BP, Spiegel A, et al. IL-1ß inflammatory response driven by primary breast cancer prevents metastasisinitiating cell colonization. Nat Cell Biol 2018;20:1084-97.

8 Voloshin T, Alishekevitz D, Kaneti L, et al. Blocking IL1 $\beta$ pathway following paclitaxel chemotherapy slightly inhibits primary tumor growth but promotes spontaneous metastasis. Mol Cancer Ther 2015;14:1385-94.

9 Coffelt SB, Kersten K, Doornebal CW, et al. IL-17-producing gammadelta $T$ cells and neutrophils conspire to promote breast cancer metastasis. Nature 2015;522:345-8.

10 Liu Y, Gu Y, Han Y, et al. Tumor exosomal RNAs promote lung premetastatic niche formation by activating alveolar epithelial TLR3 to recruit neutrophils. Cancer Cell 2016;30:243-56.

11 Spiegel A, Brooks MW, Houshyar S, et al. Neutrophils suppress intraluminal NK cell-mediated tumor cell clearance and enhance extravasation of disseminated carcinoma cells. Cancer Discov 2016;6:630-49.

12 Wculek SK, Malanchi I. Neutrophils support lung colonization of metastasis-initiating breast cancer cells. Nature 2015;528:413-7.

13 Granot Z, Henke E, Comen EA, et al. Tumor entrained neutrophils inhibit seeding in the premetastatic lung. Cancer Cell 2011:20:300-14.

$14 \mathrm{Ng} \mathrm{LG}$, Ostuni R, Hidalgo A. Heterogeneity of neutrophils. Nat Rev Immunol 2019;19:255-65.

15 Fridlender ZG, Sun J, Kim S, et al. Polarization of tumor-associated neutrophil phenotype by TGF-beta: "N1" versus "N2" TAN. Cancer Cell 2009;16:183-94

16 Sagiv JY, Michaeli J, Assi S, et al. Phenotypic diversity and plasticity in circulating neutrophil subpopulations in cancer. Cell Rep 2015;10:562-73.

17 Brinkmann V, Reichard U, Goosmann C, et al. Neutrophil extracellular traps kill bacteria. Science 2004;303:1532-5.

18 Jorch SK, Kubes P. An emerging role for neutrophil extracellular traps in noninfectious disease. Nat Med 2017;23:279-87.

19 Arelaki S, Arampatzioglou A, Kambas K, et al. Gradient infiltration of neutrophil extracellular traps in colon cancer and evidence for their involvement in tumour growth. PLoS One 2016;11:e0154484.

20 Albrengues J, Shields MA, Ng D, et al. Neutrophil extracellular traps produced during inflammation awaken dormant cancer cells in mice. Science 2018;361 doi:10.1126/science.aao4227

21 Lee W, Ko SY, Mohamed MS, et al. Neutrophils facilitate ovarian cancer premetastatic niche formation in the omentum. $J$ Exp Med 2019;216:176-94.

22 van der Windt DJ, Sud V, Zhang H, et al. Neutrophil extracellular traps promote inflammation and development of hepatocellular carcinoma in nonalcoholic steatohepatitis. Hepatology 2018;68:1347-60.

23 Demers M, Krause DS, Schatzberg D, et al. Cancers predispose neutrophils to release extracellular DNA traps that contribute to cancer-associated thrombosis. Proc Natl Acad Sci U S A 2012;109:13076-81.

24 Pieterse E, Rother N, Garsen M, et al. Neutrophil extracellular traps drive endothelial-to-mesenchymal transition. Arterioscler Thromb Vasc Biol 2017;37:1371-9.

25 Hisada Y, Grover SP, Maqsood A, et al. Neutrophils and neutrophil extracellular traps enhance venous thrombosis in mice bearing human pancreatic tumors. Haematologica 2020;105:218-25.

26 Zucker-Franklin D. Electron microscopic studies of human granulocytes: structural variations related to function. Semin Hematol 1968;5:109-33.

27 Pliyev BK, Ivanova AV, Savchenko VG. Extracellular NAD(+) inhibits human neutrophil apoptosis. Apoptosis 2014;19:581-93.

28 El Kebir D, de Oliveira Lima Dos Santos E, Mansouri S, et al. Mild acidosis delays neutrophil apoptosis via multiple signaling pathways and acts in concert with inflammatory mediators. J Leukoc Biol 2017;102:1389-400. doi:10.1189/jlb.3A0117-041R

29 Yang X, Li C, Ng KT-P, et al. II-17A exacerbates hepatic ischemiareperfusion injury in fatty liver by promoting neutrophil infiltration and mitochondria-driven apoptosis. J Leukoc Biol 2020;108:1603-13.

30 Rice CM, Davies LC, Subleski JJ, et al. Tumour-elicited neutrophils engage mitochondrial metabolism to circumvent nutrient limitations and maintain immune suppression. Nat Commun 2018;9:5099. 
31 Tanimura A, Miyoshi K, Horiguchi T, et al. Mitochondrial activity and unfolded protein response are required for neutrophil differentiation. Cell Physiol Biochem 2018;47:1936-50.

32 Chen C-Y, Tsai Y-F, Huang W-J, et al. Propofol inhibits endogenous formyl peptide-induced neutrophil activation and alleviates lung injury. Free Radic Biol Med 2018;129:372-82.

33 Marques PE, Amaral SS, Pires DA, et al. Chemokines and mitochondrial products activate neutrophils to amplify organ injury during mouse acute liver failure. Hepatology 2012;56:1971-82.

34 Bao Y, Ledderose C, Seier T, et al. Mitochondria regulate neutrophil activation by generating ATP for autocrine purinergic signaling. J Biol Chem 2014;289:26794-803.

35 Zhou W, Cao L, Jeffries J, et al. Neutrophil-specific knockout demonstrates a role for mitochondria in regulating neutrophil motility in zebrafish. Dis Model Mech 2018;11 doi:10.1242/dmm.033027

36 Zheng X, Chen M, Meng X, et al. Phosphorylation of dynamin-related protein 1 at Ser616 regulates mitochondrial fission and is involved in mitochondrial calcium uniporter-mediated neutrophil polarization and chemotaxis. Mol Immunol 2017;87:23-32.

37 Lood C, Blanco LP, Purmalek MM, et al. Neutrophil extracellular traps enriched in oxidized mitochondrial DNA are interferogenic and contribute to lupus-like disease. Nat Med 2016;22:146-53.

38 Boilard E, Fortin PR. Connective tissue diseases: mitochondria drive NETosis and inflammation in SLE. Nat Rev Rheumatol 2016;12:195-6.

39 Casanova-Acebes M, Pitaval C, Weiss LA, et al. Rhythmic modulation of the hematopoietic niche through neutrophil clearance. Cell 2013;153:1025-35.

40 Bronte V, Brandau S, Chen S-H, et al. Recommendations for myeloid-derived suppressor cell nomenclature and characterization standards. Nat Commun 2016;7:12150.

41 Milne JC, Lambert PD, Schenk S, et al. Small molecule activators of SIRT1 as therapeutics for the treatment of type 2 diabetes. Nature 2007;450:712-6.

42 Subramanian G, Chaudhury P, Malu K, et al. Lamin B receptor regulates the growth and maturation of myeloid progenitors via its sterol reductase domain: implications for cholesterol biosynthesis in regulating myelopoiesis. J Immunol 2012;188:85-102.

43 Malu K, Garhwal R, Pelletier MGH, et al. Cooperative activity of GABP with PU.1 or C/EBPE regulates lamin B receptor gene expression, implicating their roles in granulocyte nuclear maturation. J Immunol 2016;197:910-22.

44 Zhang Y, Yao Y, Qiu X, et al. Listeria hijacks host mitophagy through a novel mitophagy receptor to evade killing. Nat Immunol 2019;20:433-46.
45 Sørensen OE, Borregaard N. Neutrophil extracellular traps - the dark side of neutrophils. J Clin Invest 2016;126:1612-20.

46 Yu C-H, Davidson S, Harapas CR, et al. TDP-43 triggers mitochondrial DNA release via MPTP to activate cGAS/STING in ALS. Cell 2020;183:636-49.

47 Chalkiadaki A, Guarente L. The multifaceted functions of sirtuins in cancer. Nat Rev Cancer 2015;15:608-24.

48 Grolla AA, Torretta S, Gnemmi I, et al. Nicotinamide phosphoribosyltransferase (NAMPT/PBEF/visfatin) is a tumoural cytokine released from melanoma. Pigment Cell Melanoma Res 2015;28:718-29.

49 Mascarell L, Truffa-Bachi P. T lymphocyte activation initiates the degradation of the CD62L encoding mRNA and increases the transcription of the corresponding gene. Immunol Lett 2004:94:115-22.

50 Rankin SM. The bone marrow: a site of neutrophil clearance. J Leukoc Biol 2010;88:241-51.

51 Uhl B, Vadlau Y, Zuchtriegel G, et al. Aged neutrophils contribute to the first line of defense in the acute inflammatory response. Blood 2016;128:2327-37.

52 Zhang D, Chen G, Manwani D, et al. Neutrophil ageing is regulated by the microbiome. Nature 2015;525:528-32.

$53 \mathrm{Kim} \mathrm{JH}$, Podstawka J, Lou Y, et al. Aged polymorphonuclear leukocytes cause fibrotic interstitial lung disease in the absence of regulation by B cells. Nat Immunol 2018;19:192-201.

54 Carvalho LO, Aquino EN, Neves ACD, et al. The neutrophil nucleus and its role in neutrophilic function. J Cell Biochem 2015;116:1831-6.

55 Manley HR, Keightley MC, Lieschke GJ. The neutrophil nucleus: an important influence on neutrophil migration and function. Front Immunol 2018;9:2867.

56 Chen X, Lu Y, Zhang Z, et al. Intercellular interplay between SIRT1 signalling and cell metabolism in immune cell biology. Immunology 2015;145:455-67.

57 Rodríguez-Espinosa O, Rojas-Espinosa O, Moreno-Altamirano $\mathrm{MMB}$, et al. Metabolic requirements for neutrophil extracellular traps formation. Immunology 2015;145:213-24.

58 Yang L, Liu Q, Zhang X, et al. DNA of neutrophil extracellular traps promotes cancer metastasis via CCDC25. Nature 2020;583:133-8.

59 Park IH, Kong S-Y, Ro JY, et al. Prognostic implications of tumorinfiltrating lymphocytes in association with programmed death ligand 1 expression in early-stage breast cancer. Clin Breast Cancer 2016;16:51-8. 


\section{Correction: Aged neutrophils form mitochondria-dependent vital NETs to promote breast cancer lung metastasis}

Yang C, Wang Z, Li L, et al. Aged neutrophils form mitochondria-dependent vital NETs to promote breast cancer lung metastasis. J Immunother Cancer 2021;9:e002875. doi:10.1136/jitc-2021-002875.

This article has been corrected since it was first published. Affiliations 2, 3 and 4 were listed in the wrong order and have now been rearranged accordingly.

Open access This is an open access article distributed in accordance with the Creative Commons Attribution Non Commercial (CC BY-NC 4.0) license, which permits others to distribute, remix, adapt, build upon this work non-commercially, and license their derivative works on different terms, provided the original work is properly cited, appropriate credit is given, any changes made indicated, and the use is non-commercial. See http://creativecommons.org/licenses/by-nc/4.0/.

(C) Author(s) (or their employer(s)) 2021. Re-use permitted under CC BY-NC. No commercial re-use. See rights and permissions. Published by BMJ.

J Immunother Cancer 2021;9:e002875corr1. doi:10.1136/jitc-2021-002875corr1

A) Check for updates 